UNIVERSIDADE DE SÃO PAULO

ESCOLA DE ARTES, CIÊNCIAS E HUMANIDADES

PROGRAMA DE PÓS-GRADUAÇÃO EM MODELAGEM DE SISTEMAS

COMPLEXOS

VICTOR CAMARGO

Análise da Função Mestra de Estabilidade em um Modelo de Ciclos Econômicos

São Paulo 


\section{Análise da Função Mestra de Estabilidade em um Modelo de Ciclos} Econômicos

Dissertação apresentada à Escola de Artes, Ciências e Humanidades da Universidade de São Paulo para obtenção do título de Mestre em Ciências pelo Programa de Pós-graduação em Modelagem de Sistemas Complexos.

Área de concentração: Redes Complexas e Sistemas Dinâmicos

Orientador: Prof. Dr. Fernando Fagundes Ferreira

São Paulo 


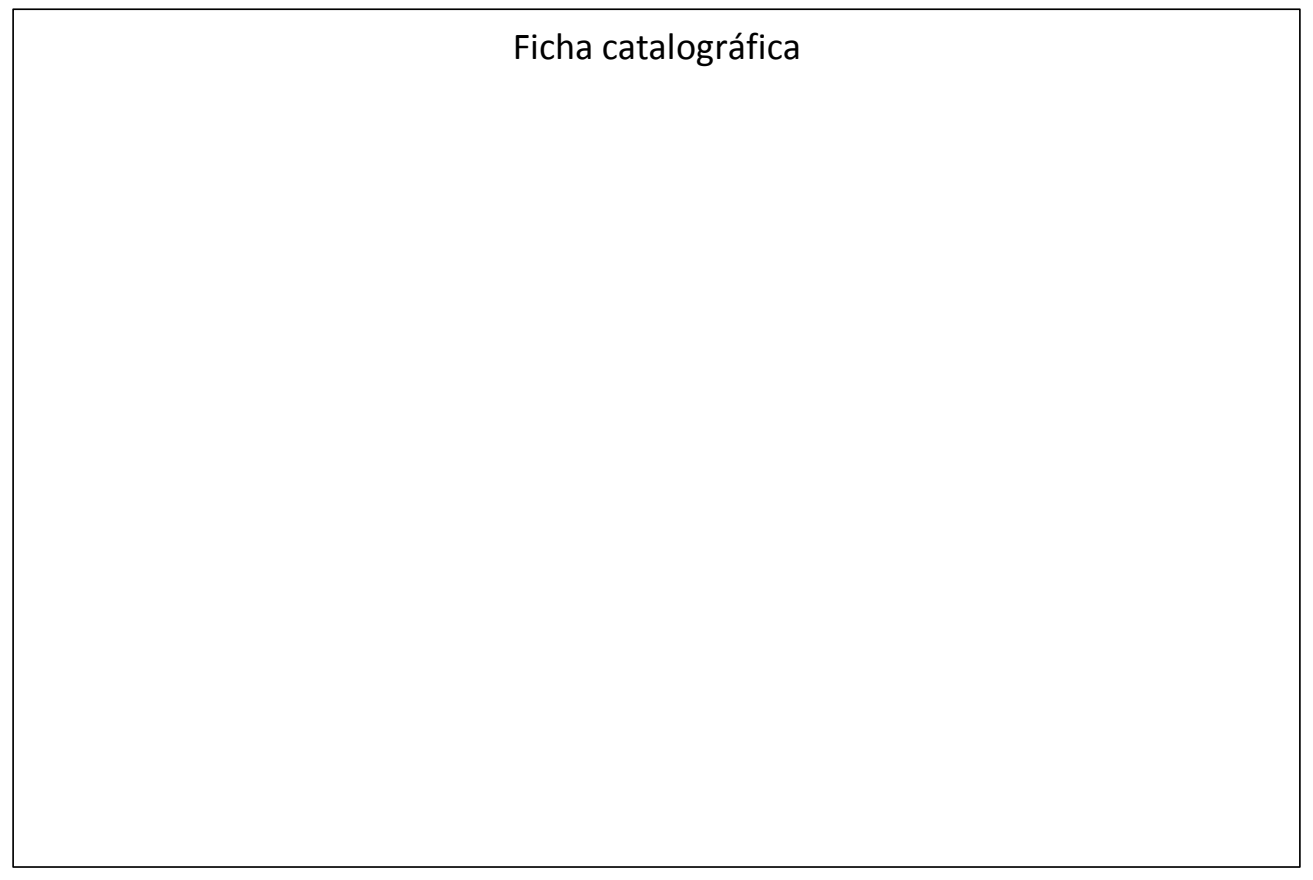


Dissertação de autoria de Victor Emmanuel Camargo Molina, sob o título "Análise da Função Mestra de Estabilidade em um Modelo de Ciclos Econômicos", apresentada à Escola de Artes, Ciências e Humanidades da Universidade de São Paulo, para obtenção do título de Mestre em Ciências pelo Programa de Pós-graduação em Modelagem de Sistemas Complexos, na área de concentração Redes Complexas e Sistemas Dinâmicos, aprovada em de de pela comissão julgadora constituída pelos doutores:

Prof. Dr.

Instituição:

Presidente

Prof. Dr.

Instituição:

Prof. Dr.

Instituição:

Prof. Dr.

Instituição: 


\section{Agradecimentos}

Quero agradecer em primeiro lugar ao Glorioso Pai Criador, causa abscondita do conhecido.

Aos meus pais, Victor Hugo e Roraima, que incutiram em mim todos os dias amor, sabedoria, coragem, paciência, força, dedicação,... e um interminável. Eles foram e serão meus heróis! assim como minhas irmãzinhas Valéria e Victoria, que me ensinam muito e me enchem de alegria!

Ao meu orientador Prof. Dr. Fernando Fegundes Ferreira, que tem sido um apoio incondicional desde que iniciei o mestrado como professor e como amigo, me dando uma oportunidade de crescer academicamente e como pessoa, compartilhando seus conhecimentos, experiências e dedicação para realizar esta dissertação.

Ao meu amigo Amaury, sua esposa Eloisa e os filhos Paulo e Marcia, que me deram seu apoio e amizade durante toda essa experiência em São Paulo.

... e a todos aqueles que direta ou indiretamente contribuíram com seu grão de areia para alcançar esse momento, OBRIGADO! 


\section{Resumo}

Camargo Molina, Victor Emmanuel. Título do trabalho: Análise da Função Mestra de Estabilidade em um Modelo de Ciclos Econômicos. 2020. 57 f. Dissertação (Mestrado em Ciências) - Escola de Artes, Ciências e Humanidades, Universidade de São Paulo, São Paulo, 2020.

Neste trabalho, estudamos a estabilidade dos estados sincronizados por meio da Master Stability Function (MSF), para uma rede de osciladores caóticos de Van Der Pol. Estudamos a interação entre sistemas dinâmicos não lineares que representam uma macroeconomia simplificada. O modelo é uma versão modificada e estendida do oscilador de Van der Pol. Considerou-se apenas três variáveis de estados: a entrada de capital estrangeiro, a poupança das famílias e o produto interno bruto. Com efeito, analisamos possíveis padrões que resultam desta dinâmica bem como mudanças estruturais quando se varia o fluxo de capital estrangeiro, escolhido aqui como o parâmetro de controle. Há uma riqueza de comportamentos dinâmicos. Para certos valores do parâmetro de controle ocorre a sincronização entre as economias. Contudo, elas podem ser instáveis. Assim, aplicamos o método da função mestra de estabilidade (MSF) para avaliar a estabilidade. O resultado principal foi observar a ocorrência de sincronizações instáveis quando o acoplamento foi unidirecional, e estável no caso bidirecional. Além disso, o método de análise permite que se explore um número arbitrário de economias acopladas com diferentes topologias de redes.

Palavras-chaves: Rede complexa, Sincronização, Função Mestra de Estabilidade, Oscilador de Van der Pol. 


\begin{abstract}
Camargo Molina, Victor Emmanuel. Work title: Master Stability Function Analysis in an Economic Cycle Model. 2020. 57 p. Dissertation (Master of Science) - School of Arts, Sciences and Humanities, University of São Paulo, São Paulo, 2020.

In the present work, we study the stability of synchronized states through the Master Stability Function (MSF) for a Van Der Pol oscillator network. First, we study the interaction between nonlinear dynamic systems that represent a simplified macroeconomie. The model is a modified and extended version of the Van der Pol oscillator, where three state variables were considered: foreign capital inflows, household savings and gross domestic product. Indeed, we analyze possible patterns that result from this dynamics as well as structural changes when varying the foreign capital flow, chosen here as the control parameter. There is a wealth of dynamic behaviors. For certain control parameter values synchronization occurs between the economies. However, they may be unstable. Thus, we apply the Master Stability Function method to evaluate stability. The main result was to observe the occurrence of unstable synchronizations when the coupling was unidirectional, and stable in the bidirectional case. In addition, the analysis method allows us to explore an arbitrary number of macroeconomies coupled with different network topologies.
\end{abstract}

Keywords: Complex Network, Synchronization, Master Stability Function, Van der Pol Oscillator. 


\section{Lista de figuras}

Figura 1 - Esquema representando um conjunto de $i(i=1,2, \ldots, N)$ osciladores isolados no espaço, cuja dinâmica individual é dada pela Eq. 3. Fonte: Elaborado pelo autor. . . . . . . . . . . . . . . . 1

Figura 2 - Esquema representando uma rede de $N=2$ osciladores no espaço, cuja interação é dada pela Eq. 6. Fonte: Elaborado pelo autor. . . . . . . . . . 13

Figura 3 - Esquema representando uma rede do tipo anel de $N$ osciladores no espaço, onde, aumentado o parâmetro de acoplamento $\varepsilon$, todos os elementos no sistema sincronizam, convergindo para uma solução que corresponde à dinâmica de um oscilador isolado Eq. 9 no subespaço da variedade de sincronismo idêntico. Fonte: Elaborado pelo autor. . . . . 1

Figura 4 - Atrator tridimensional das variáveis $x, y, z$, associado ao sistema Eq. 32 para os seguintes parâmetros: $(m, p, d, c, s, r)=(0.02,0.4,1.0,50,10,0.1)$ respectivamente. Fonte: Elaborado pelo autor. . . . . . . . . . . . 23

Figura 5 - Projeções $x y$ e $x z$ do atrator tridimensional associado ao sistema Eq. 32 para os seguintes parâmetros: $(m, p, d, c, s, r)=(0.02,0.4,1.0,50,10,0.1)$ respectivamente. Fonte: Elaborado pelo autor. . . . . . . . . . . . 24

Figura 6 - Projeções $z x$ dos atratores do sistema Eq. (39) para diferentes "feedbacks"; $(a) \xi=0.0$ e $(b) \xi=1.0$; com os seguintes parâmetros: $(m, p, d, c, s, r)=(0.02,0.4,1.0,50,10,0.1)$ respectivamente. Os cálculos foram feitos com $5 \times 10^{4}$ iterações, ignorando $4 \times 10^{4}$ transientes e, portanto, considerando $1 \times 10^{4}$ iterados após os transientes. Fonte: Elaborado pelo autor.

Figura 7 - Atrator do sistema Eq. 39 para diferentes "feedbacks", e com condições iniciais positivas $x(0), y(0), z(0)=0.05,0.20,0.02$ e negativas $x(0), y(0), z(0)=$ $-0.05,-0.20,-0.02$; temos que a) $\xi=0.0$ com condições iniciais positivas, $b) \xi=0.0$ com condições iniciais negativas, $c) \xi=1.0 \mathrm{com}$ condições iniciais positivas e $d$ ) $\xi=1.0$ com condições iniciais negativas; com os seguintes parâmetros: $(m, p, d, c, s, r)=(0.02,0.4,1.0,50,10,0.1)$ respectivamente. . . . . . . . . . . . . . . . . 26 
Figura 8 - Espectro dos Maiores Expoentes de Lyapunov $\lambda_{1}, \lambda_{2}$ e $\lambda_{3}$ em função do parâmetro de controle $c$ para o sistema Eq. (39) com uma intensidade de feedback de $\xi=1.0$ e com $(m, p, d, c, s, r)=(0.02,0.4,1.0,50,10,0.1)$. Fonte: Elaborado pelo autor. . . . . . . . . . . . . . . . . . . . . 28

Figura 9 - Dimensão Kaplan-Yorke como função do parâmetro de controle $c$ para o sistema Eq. 39 com uma intensidade de feedback de $\xi=1.0$ e com $(m, p, d, c, s, r)=(0.02,0.4,1.0,50,10,0.1)$. Fonte: Elaborado pelo autor. 30

Figura 10 - Espectro dos Maiores Expoentes de Lyapunov $\lambda_{1}, \lambda_{2}$ e $\lambda_{3}$ em função do parâmetro de acoplamento $\xi$ para o sistema Eq. 39; $\operatorname{com}(m, p, d, c, s, r)=$ $(0.02,0.4,1.0,50,10,0.1)$. Fonte: Elaborado pelo autor. . . . . . . . .

Figura 11 - Dimensão Kaplan-Yorke como função do parâmetro de acoplamento $\xi$ para o sistema Eq. 39; com $(m, p, d, c, s, r)=(0.02,0.4,1.0,50,10,0.1)$. Fonte: Elaborado pelo autor. . . . . . . . . . . . . . . . 32

Figura 12 - Diagrama de sincronização do sistema Eq. (38) para uma rede de $N=2$ nós em função do parâmetro de acoplamento $\xi$ associado aos estados $x_{i}, y_{i}, z_{i}$ para diferentes condições iniciais em cada iteração temporal; com $(m, p, d, c, s, r)=(0.02,0.4,1.0,50,10,0.1) ;$ acoplamento do tipo unidirecional. Os cálculos foram feitos com $5 \times 10^{4}$ iterações, ignorando $4 \times 10^{4}$ transientes e, portanto, considerando $1 \times 10^{4}$ iterados após os transientes. Fonte: Elaborado pelo autor. . . . . . . . . . . . 36

Figura 13 - Diagrama de sincronização do sistema Eq. (38) para uma rede de $N=2$ nós em função do parâmetro de acoplamento $\xi$ associado aos estados $x_{i}, y_{i}, z_{i}$ para a mesma condição inicial em cada iteração temporal; com $(m, p, d, c, s, r)=(0.02,0.4,1.0,50,10,0.1)$; acoplamento do tipo unidirecional. Os cálculos foram feitos com $5 \times 10^{4}$ iterações, ignorando $4 \times 10^{4}$ transientes e, portanto, considerando $1 \times 10^{4}$ iterados após os transientes. Fonte: Elaborado pelo autor. . . . . . . . . . . . . . 38 
Figura 14 - Diagrama de sincronização do sistema Eq. (38) para uma rede de $N=2$ nós em função do parâmetro de acoplamento $\xi$ associado aos estados $x_{i}, y_{i}, z_{i}$ para diferentes condições iniciais em cada iteração temporal; com $(m, p, d, c, s, r)=(0.02,0.4,1.0,50,10,0.1) ;$ acoplamento do tipo bidirecional. Os cálculos foram feitos com $5 \times 10^{4}$ iterações, ignorando $4 \times 10^{4}$ transientes e, portanto, considerando $1 \times 10^{4}$ iterados após os transientes. Fonte: Elaborado pelo autor. . . . . . . . . . . . . . . . . . 40

Figura 15 - Diagrama de sincronização do sistema Eq. (38) para uma rede de $N=2$ nós em função do parâmetro de acoplamento $\xi$ associado aos estados $x_{i}, y_{i}, z_{i}$ para a mesma condição inicial em cada iteração temporal; com $(m, p, d, c, s, r)=(0.02,0.4,1.0,50,10,0.1)$; acoplamento do tipo bidirecional. Os cálculos foram feitos com $5 \times 10^{4}$ iterações, ignorando $4 \times 10^{4}$ transientes e, portanto, considerando $1 \times 10^{4}$ iterados após os transientes. Fonte: Elaborado pelo autor. . . . . . . . . . . . . . . . . . 41

Figura 16 - Função Mestra de Estabilidade $\lambda_{T}$ em função do parâmetro de acoplamento $\xi$ para o sistema Eq. 38 com uma configuração unidireccional; fixando os valores $(N, m, p, d, c, s, r)=(2,0.02,0.4,1.0,50,10,0.1)$. Fonte: Elaborado pelo autor.

Figura 17 - Função Mestra de Estabilidade $\lambda_{T}$ sob vários esquemas de acoplamento $\xi$ para o sistema Eq. 38 com uma configuração unidireccional; fixando os valores $(N, m, p, d, c, s, r)=(2,0.02,0.4,1.0,50,10,0.1)$. A notação $x, y, z \rightarrow x, y, z$, indica o acoplamento como sendo dos componentes $x, y, z$ de um oscilador para os componentes $x, y, z$ de outro oscilador.Fonte: Elaborado pelo autor. . . . . . . . . . . . . . . . . . 46

Figura 18 - Função Mestra de Estabilidade $\lambda_{T}$ em função do parâmetro de acoplamento $\xi$ para o sistema Eq. 38 com uma configuração bidireccional; fixando os valores $(N, m, p, d, c, s, r)=(2,0.02,0.4,1.0,50,10,0.1)$. Fonte: Elaborado pelo autor.

Figura 19 - Função Mestra de Estabilidade $\lambda_{T}$ sob vários esquemas de acoplamento $\xi$ para o sistema Eq. 38; com $(N, m, p, d, c, s, r)=(2,0.02,0.4,1.0,50,10,0.1)$. A notação $x, y, z \rightarrow x, y, z$, indica o acoplamento como sendo dos componentes $x, y, z$ de um oscilador para os componentes $x, y, z$ de outro oscilador.Fonte: Elaborado pelo autor. 
Figura 20 - Função Mestra de Estabilidade $\lambda_{T}$ acoplada por meio de $\xi$ pelos diferentes componentes $z \rightarrow z, y, z \rightarrow y, z$ e $x, y, z \rightarrow x, y, z$ para o sistema Eq. 38; com $(N, m, p, d, c, s, r)=(2,0.02,0.4,1.0,50,10,0.1)$. Fonte: Elaborado pelo autor. . . . . . . . . . . . . . . . . . . . 51 


\section{Sumário}

Introdução . . . . . . . . . . . . . . . . . . . 9 9

2 Função Mestra de Estabilidade . . . . . . . . . . . . 12

2.1 Formalismo da Função Mestra de Estabilidade . . . . . . . . . 12

$2.2 \quad$ Estabilidade linear . . . . . . . . . . . . . . . . . . . . 15

$3 \quad$ Oscilador de Van der Pol . . . . . . . . . . . . . . . 19

$3.1 \quad$ Modelo Macroeconômico . . . . . . . . . . . . . . . . . . . . 19

$3.2 \quad$ Sistema Macroeconômico Acoplado . . . . . . . . . . . . . . 21

3.3 Comportamentos Dinâmicos . . . . . . . . . . . . . . 22

3.3.1 Atrator do Sistema . . . . . . . . . . . . . . . 22

3.3.2 Expoentes de Lyapunov e Dimensão Fractal . . . . . . . . . 27

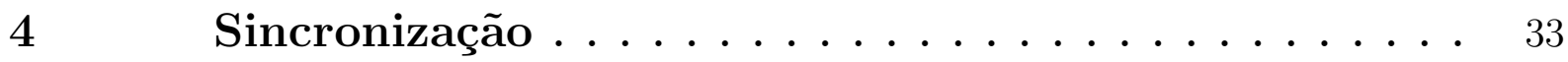

4.1 Caracterização de Estados Sincronizados . . . . . . . . . . 34

4.2 Estados de Sincronização . . . . . . . . . . . . . 35

4.3 Análise de Estabilidade de Estados Sincronizados . . . . . . . 42

4.3.1 Função Mestra de Estabilidade . . . . . . . . . . . . . . . 42

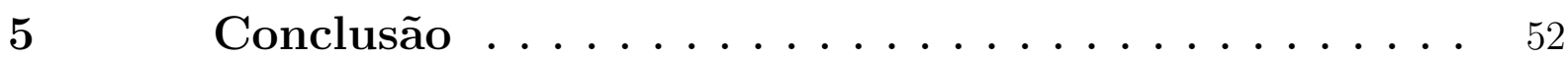

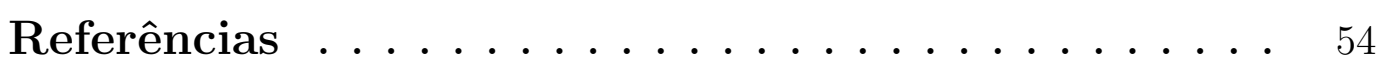

Apêndice A-Runge-Kutta 4to ordem . . . . . . 57 


\section{Introdução}

Com origem no trabalho realizado por Louis Pecora e Thomas Carroll em 1989 (PECORA; CARROLL, 1990), onde eles descreveram o comportamento de um sistema acoplado ou vinculado por meio de suas variáveis de estado ou sinais. Assim, quando os sinais dos expoentes de Lyapunov de tais variáveis de estado são negativos, o sistema sincroniza, ou seja, todas as trajetórias convergem para o mesmo valor. Nesse primeiro trabalho, eles se referiram a essa ferramenta como sub-Expoentes de Lyapunov, que foi posteriormente alterada para Expoentes de Lyapunov condicionais em seu trabalho intitulado "Driving Systems With Chaotic Signals" (PECORA; CARROLL, 1991). O formalismo da Função Mestra de Estabilidade foi desenvolvido alguns anos depois por Louis Pecora e Thomas Carroll, onde eles se focaram em mostrar de uma maneira mais geral a estabilidade dos estados totalmente sincronizados de topologias de redes mais complexas, além de tentar entender as propriedades subjacentes da estrutura da rede. Um dos pontos mais proeminentes ao utilizar esta técnica é que é possível deixar de lado as características ou particularidades dos osciladores, e permite caracterizar a estabilidade global dos estados sincronizados ou qual a mesma sincronizabilidade da rede, revelando assim detalhes importantes na relação estrutura-dinâmica da rede.

Para capturar a essência da Função Mestra de Estabilidade, um sistema dinâmico deve ser considerado, o qual é uma rede de $N$ osciladores idênticos acoplados, na qual seus parâmetros devem ser escolhidos de tal forma que exibam uma dinâmica caótica na sincronização. Tal formalismo pode ser aplicado em sistemas de tempo contínuo e sistemas de tempo discretos. Quando todos os osciladores são inicialmente definidos para a variedade de sincronização, esses estados permanecerão sincronizados. Agora, está claro que uma das principais questões que surge é se a variedade de sincronização é estável quando uma pequena perturbação é aplicada no sistema, portanto estamos interessados em estudar como é a separação das trajetórias infinitesimais da equação variacional do sistema, que é solução na sincronização. Calculando o máximo expoente de Lyapunov dessa equação variacional na solução de sincronização, obtemos o expoente de Lyapunov transversal relacionado à órbita do sistema que é conhecida como a Função Mestra de Estabilidade. 
Se a Função Mestra de Estabilidade for negativa, uma pequena perturbação do estado de sincronização diminuirá exponencialmente, portanto a solução síncrona é estável. Por outro lado, se for positivo, a solução de sincronização é instável e pode ser fisicamente difícil de analisar porque as trajetórias divergem do estado de sincronização.

A Função Mestra de Estabilidade apresenta estabilidade local, mesmo que seja negativa ou muito próxima de zero, pode-se observar que o sistema se comporta de maneira fora de sincronia, pois dependerá sempre das propriedades do atrator caótico. Em geral, as propriedades dessa técnica são invariantes sob pequenas variações de parâmetros do sistema. A vantagem fornecida pela estrutura da Função Mestra de Estabilidade é que ela oferece um critério matematicamente sólido-objetivo e sem se referir diretamente às propriedades dos osciladores. No capítulo 2, a partir da análise de um sistema dinâmico complexo formado por uma rede de $N$ osciladores, introduzimos o formalismo matemático da função mestra de estabilidade.

Por outra parte sabemos que a economia afeta a vida de bilhões de pessoas que, por falhas de mercado, sofrem devido ao desemprego, crescimento do produto interno bruto baixo, concentração de renda e pobreza. Um dos grandes problemas é a dificuldade de prever e previnir as crises. De modo geral, a previsibilidade em sistemas econômicos representa um desafio para diversos campos de estudo. Estes sistemas são formados por um elevado número de agentes heterogêneos que interagem de forma não linear no nível microscópico, que reagem às informações de indicadores macroecônomicos (feedbacks) e ainda sofrem influências de outras economias pelo fluxo de capital entre elas. A quantidade de parâmetros envolvidos, a não linearidade e a dificuldade de capturar via modelagem as expectativas dos agentes estão na origem do problema. Por outro lado, nossa incapacidade presente de produzir bons modelos deixam muitas perguntas em aberto. Não somos capazes de evitar crises econômicas e controlar o risco sistêmico causado pela propagação dos efeitos de uma recessão ou crash de um país sobre seus parceiros. Assim, pode-se dizer que é importante estudar modelos simplificados para entender os mecanismos fundamentais dos padrões coletivos de economias acopladas.

No presente trabalho examinamos o comportamento dinâmico entre economias que são idênticas, as quais foram acopladas por meio da variável troca de capital. No capítulo 3, apresentamos um modelo tridimensional de Van Der Pol, onde modelamos uma macroeconomia simplificada. Estas economias foram aproximadas por um modelo de oscilador de ciclo-limite. O modelo será descrito na próxima seção, contudo podemos dizer 
que ele é uma modificação do sistema bidimensional de Van der Pol (POL, 1926; BOUALI, 1999) com a adição de um termo de feedback que representa as trocas internacionais. Além disso, do ponto de vista econômico, o sistema simula a fuga de capitais observada nos países menos desenvolvidos. Então, neste capítulo examinamos o efeito da entrada e saída de capital entre dois sistemas econômicos conjugados. 


\section{Função Mestra de Estabilidade}

\subsection{Formalismo da Função Mestra de Estabilidade}

Para entrar no formalismo da Função Mestra de Estabilidade, procederemos à análise de um sistema dinâmico complexo que consiste em uma rede de osciladores caóticos de tempo contínuo acoplados. Para descrever essa rede, consideramos um conjunto de $N$ nós idênticos, onde o vetor $\boldsymbol{\psi}_{i}(t)$ de dimensão $l \times 1$ da forma

$$
\boldsymbol{\psi}_{i}(t)=\left[\begin{array}{c}
\psi_{i}^{1}(t) \\
\psi_{i}^{2}(t) \\
\psi_{i}^{3}(t) \\
\vdots \\
\psi_{i}^{l}(t)
\end{array}\right]
$$

representa o estado do elemento $i$-ésimo $(i=1,2, \ldots, N)$ da rede em um passo contínuo de tempo $t$, e $\psi_{i}^{1}(t), \psi_{i}^{2}(t), \psi_{i}^{3}(t), \ldots, \psi_{i}^{l}(t)$, as variáveis de estado que o compõem.
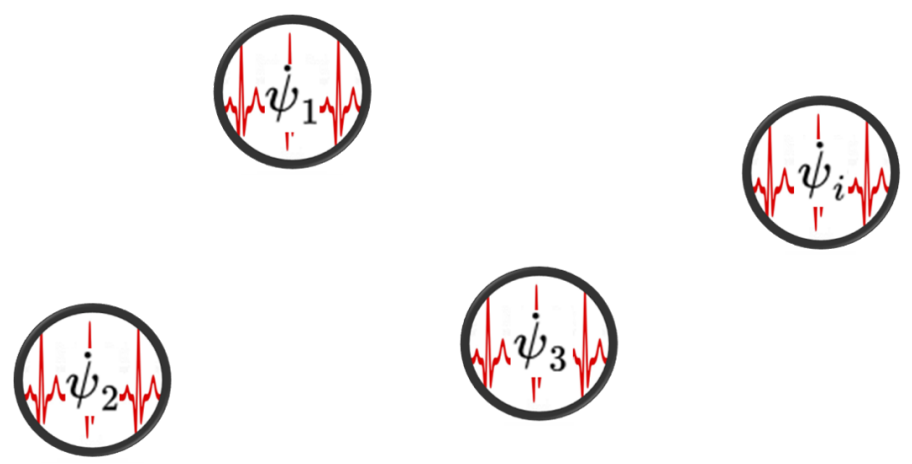

Figura 1 - Esquema representando um conjunto de $i(i=1,2, \ldots, N)$ osciladores isolados no espaço, cuja dinâmica individual é dada pela Eq. 3. Fonte: Elaborado pelo autor.

Agora, a dinâmica de um oscilador completamente isolado no espaço (Fig. 1) é descrita por

$$
\mathbf{f}\left(\boldsymbol{\psi}_{i}(t)\right)=\left[\begin{array}{c}
f_{1}\left(\boldsymbol{\psi}_{i}(t)\right) \\
f_{2}\left(\boldsymbol{\psi}_{i}(t)\right) \\
f_{3}\left(\boldsymbol{\psi}_{i}(t)\right) \\
\vdots \\
f_{l}\left(\boldsymbol{\psi}_{i}(t)\right)
\end{array}\right]
$$


onde a dinâmica das variáveis de estado é governada de acordo com as funções $f_{1}\left(\boldsymbol{\psi}_{i}(t)\right)$, $f_{2}\left(\boldsymbol{\psi}_{i}(t)\right), f_{3}\left(\boldsymbol{\psi}_{i}(t)\right), \ldots, f_{l}\left(\boldsymbol{\psi}_{i}(t)\right)$, que compõem o campo vetorial ou o vetor velocidade do campo $\mathbf{f}\left(\boldsymbol{\psi}_{i}\right)$, então podemos escrever

$$
\frac{d \boldsymbol{\psi}_{i}(t)}{d t}=\mathbf{f}\left(\boldsymbol{\psi}_{i}(t)\right)
$$

Para conectar nossa rede, precisamos estabelecer sua topologia, para isso escrevemos a matriz de acoplamento $\mathbf{C}$ de dimensão $N \times N$, da seguinte forma

$$
\mathbf{C}=\left[\begin{array}{cccc}
c_{11} & c_{12} & \cdots & c_{1 N} \\
c_{21} & \ddots & \vdots & \vdots \\
\vdots & \cdots & \ddots & \vdots \\
c_{N 1} & \cdots & \cdots & c_{N N}
\end{array}\right]
$$

onde cada um dos elementos $c_{i j}$ é o peso da conexão da aresta $i$ para $j$, e pode ter qualquer magnitude positiva ou nula.

Por sua vez, precisamos saber o valor da saída do $j$-ésimo nó que fará parte da entrada do $i$-ésimo nó. Definimos a função $l$-dimensional de acoplamento $\mathbf{h}$ como

$$
\mathbf{h}\left(\boldsymbol{\psi}_{j}(t)\right)=\left[\begin{array}{c}
h_{1}\left(\boldsymbol{\psi}_{j}(t)\right) \\
h_{2}\left(\boldsymbol{\psi}_{j}(t)\right) \\
h_{3}\left(\boldsymbol{\psi}_{j}(t)\right) \\
\vdots \\
h_{l}\left(\boldsymbol{\psi}_{j}(t)\right)
\end{array}\right],
$$

onde, o produto da função de acoplamento $\mathbf{h}\left(\boldsymbol{\psi}_{i}(t)\right)$ com os coeficientes da matriz de acoplamento $c_{i j}$ formam o campo de interação

$$
\mathbf{g}\left(\boldsymbol{\psi}_{i}(t)\right)=\sum_{j=1}^{N} c_{i j} \mathbf{h}\left(\boldsymbol{\psi}_{j}(t)\right),
$$

cuja magnitude é modulada pelo parâmetro de acoplamento global $\varepsilon$ como mostrado na Fig. 2, e assim, a interação $\varepsilon \mathbf{g}\left(\boldsymbol{\psi}_{j}(t)\right)$ conforma o valor de entrada do $i$ - ésimo nó. 


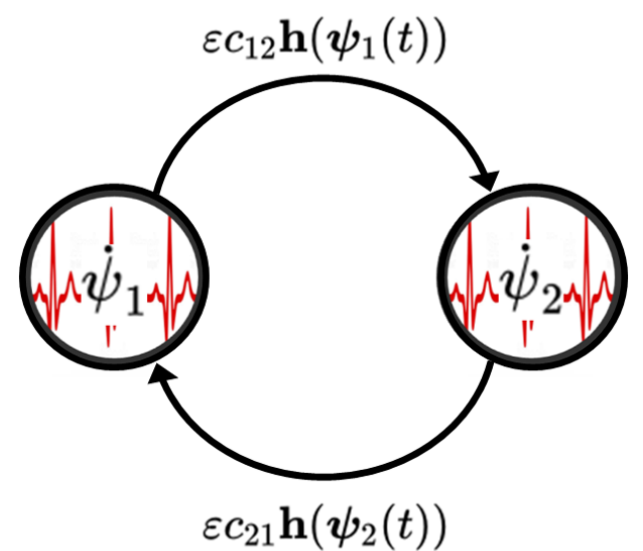

Figura 2 - Esquema representando uma rede de $N=2$ osciladores no espaço, cuja interação é dada pela Eq. 6. Fonte: Elaborado pelo autor.

Portanto, temos um conjunto de $l N$ equações dinâmicas para uma rede de osciladores acoplados, as quais são definidas como

$$
\left[\begin{array}{c}
\psi_{i}^{1}(t) \\
\psi_{i}^{2}(t) \\
\psi_{i}^{3}(t) \\
\vdots \\
\psi_{i}^{l}(t)
\end{array}\right]=\left[\begin{array}{c}
f_{1}\left(\boldsymbol{\psi}_{i}(t)\right) \\
f_{2}\left(\boldsymbol{\psi}_{i}(t)\right) \\
f_{3}\left(\boldsymbol{\psi}_{i}(t)\right) \\
\vdots \\
f_{l}\left(\boldsymbol{\psi}_{i}(t)\right)
\end{array}\right]-\varepsilon\left[\begin{array}{ccccc}
c_{11} & c_{12} & c_{13} & \cdots & c_{1 N} \\
c_{21} & \ddots & \vdots & \vdots & \vdots \\
c_{31} & \cdots & \ddots & \vdots & \vdots \\
\vdots & \cdots & \cdots & \ddots & \vdots \\
c_{N 1} & \cdots & \cdots & \cdots & c_{N N}
\end{array}\right]\left[\begin{array}{c}
h_{1}\left(\boldsymbol{\psi}_{j}(t)\right) \\
h_{2}\left(\boldsymbol{\psi}_{j}(t)\right) \\
h_{3}\left(\boldsymbol{\psi}_{j}(t)\right) \\
\vdots \\
h_{l}\left(\boldsymbol{\psi}_{j}(t)\right)
\end{array}\right],
$$

ou de uma forma geral como

$$
\frac{d \boldsymbol{\psi}_{i}(t)}{d t}=\mathbf{f}\left(\boldsymbol{\psi}_{i}(t)\right)-\varepsilon \mathbf{g}\left(\boldsymbol{\psi}_{j}(t)\right)
$$

Existe um estado induzido pelo parâmetro de acoplamento $\varepsilon$, que é completamente sincronizado nesta rede de osciladores idênticos, caracterizada por suas variáveis de estado estarem de acordo com

$$
\boldsymbol{\psi}_{1}(t)=\boldsymbol{\psi}_{2}(t)=\boldsymbol{\psi}_{3}(t)=\ldots=\boldsymbol{\psi}_{N}(t)=\boldsymbol{\psi}_{s}(t)
$$

onde $\boldsymbol{\psi}_{s}(t)$ descreve a evolução temporal de um nó isolado, bem como mostra a Fig. 3, 


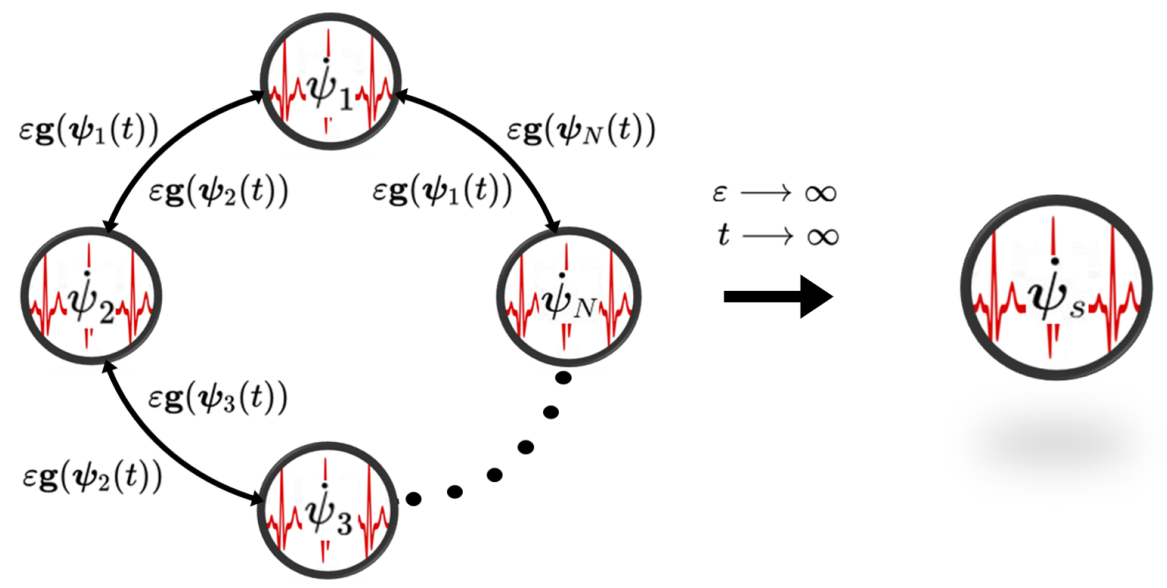

Figura 3 - Esquema representando uma rede do tipo anel de $N$ osciladores no espaço, onde, aumentado o parâmetro de acoplamento $\varepsilon$, todos os elementos no sistema sincronizam, convergindo para uma solução que corresponde à dinâmica de um oscilador isolado Eq. 9 no subespaço da variedade de sincronismo idêntico. Fonte: Elaborado pelo autor.

portanto $\boldsymbol{\psi}_{s}(t)$ é uma solução do Eq. 3, assim

$$
\frac{d \boldsymbol{\psi}_{s}(t)}{d t}=\mathbf{f}\left(\boldsymbol{\psi}_{s}(t)\right)
$$

A Eq. 8 representa um subespaço no espaço de estados em que todos os osciladores convergem para a mesma solução de um oscilador isolado Eq. 9, que pode corresponder a um atrator estranho, periódico ou estacionário. Esse subespaço onde todos os elementos são iguais é chamado pela literatura de variedade de sincronismo idêntico (HUANG et al., 2009; PECORA et al., 2000; MONTEIRO, 2010).

Uma vez que todos os $N$ osciladores estão no subespaço de sincronização ou variedade de sincronismo idêntico eles permanecerão sincronizados, portanto, duas questões importantes surgem, uma delas é: Quando o sistema converge para a variedade de sincronismo idêntico?, e outro: Essa variedade é estável na presença de pequenas perturbações?, então, sabemos que existe uma condição para a qual

$$
\boldsymbol{\psi}_{i}(t) \longrightarrow \boldsymbol{\psi}_{s}(t), \text { para } t \longrightarrow \infty
$$


portanto, consideraremos uma pequena perturbação em torno da solução para um oscilador ou nó isolado no espaço $\boldsymbol{\psi}_{s}(t)$ (Fig. 3), tal vetor de perturbação de dimensão $l \times 1$ é dado como segue

$$
\boldsymbol{\delta}_{i}(t)=\left[\begin{array}{c}
\delta_{i}^{1}(t) \\
\delta_{i}^{2}(t) \\
\delta_{i}^{3}(t) \\
\vdots \\
\delta_{i}^{l}(t)
\end{array}\right]
$$

então consideramos

$$
\boldsymbol{\psi}_{i}(t)=\boldsymbol{\psi}_{s}(t)+\boldsymbol{\delta}_{i}(t)
$$

onde podemos alcançar o estado sincronizado se

$$
\boldsymbol{\delta}_{i}(t) \longrightarrow 0, \text { para } t \longrightarrow \infty
$$

Agora que sabemos qual deve ser a condição para alcançar o variedade de sincronismo idêntico (Eq. 13), passamos a estudar a estabilidade linear em torno de $\boldsymbol{\psi}_{s}(t)$.

\section{$2.2 \quad$ Estabilidade linear}

Sabemos que a evolução linear para pequenas perturbações $\boldsymbol{\delta}_{i}(t)$ sob $\boldsymbol{\psi}_{s}(t)$ pode ser obtida pela configuração Eq. 12 na rede dos osciladores acoplados Eq. 7.

Nosso interesse é saber se tais perturbações crescem ou decaem em função do tempo, e assim, obter uma solução linear em nosso sistema de osciladores acoplados Eq. 7, devemos expandir em uma série de Taylor sobre o estado de sincronização $\boldsymbol{\psi}_{s}(t)$. Agora, a partir da Eq. 12 e Eq. 9, vamos ver que podemos escrever

$$
\frac{d \boldsymbol{\psi}_{i}(t)}{d t}=\frac{d \boldsymbol{\psi}_{s}(t)}{d t}+\frac{d \boldsymbol{\delta}_{i}(t)}{d t}=\mathbf{f}\left(\boldsymbol{\psi}_{s}(t)\right)+\frac{d \boldsymbol{\delta}_{i}(t)}{d t}
$$

obtendo consequentemente uma expressão para a evolução temporal da pequena perturbação $\boldsymbol{\delta}_{i}(t)$ escrito da seguinte maneira

$$
\frac{d \boldsymbol{\delta}_{i}(t)}{d t}=\frac{d \boldsymbol{\psi}_{i}(t)}{d t}-\mathbf{f}\left(\boldsymbol{\psi}_{s}(t)\right)
$$


Agora, vamos expandir a série de Taylor $\dot{\boldsymbol{\psi}}_{i}(t)$ sob $\boldsymbol{\psi}_{s}(t)$, onde por a Eq. 7 sabemos que é o mesmo que fazê-lo na dinâmica do sistema $\mathbf{f}\left(\boldsymbol{\psi}_{i}(t)\right)$ e sobre a função de acoplamento $\mathbf{h}\left(\boldsymbol{\psi}_{j}(t)\right)$. Temos então que

$$
\left.f_{n}\left(\boldsymbol{\psi}_{i}(t)\right) \cong f_{n}\left(\boldsymbol{\psi}_{i}(t)\right)\right|_{\boldsymbol{\psi}_{i}=\boldsymbol{\psi}_{s}}+\sum_{p=1}^{l}\left[\left.\frac{\partial f_{n}\left(\boldsymbol{\psi}_{i}(t)\right)}{\partial \psi_{i p}}\right|_{\boldsymbol{\psi}_{i}=\boldsymbol{\psi}_{s}}\right]\left[\psi_{i p}(t)-\psi_{s p}(t)\right]
$$

onde desprezamos os termos quadráticos já que consideramos que $\boldsymbol{\delta}_{i}(t)=\left|\psi_{i p}(t)-\psi_{s p}(t)\right|$ e $\left|\psi_{i p}(t)-\psi_{s p}(t)\right|<<1$, portanto, qualquer potência $m \geq 2$ de $\left|\psi_{i p}(t)-\psi_{s p}(t)\right|^{m}$ pode ser insignificante. Da mesma forma, obtemos similarmente a função de acoplamento

$$
\left.h_{n}\left(\boldsymbol{\psi}_{j}(t)\right) \cong h_{n}\left(\boldsymbol{\psi}_{j}(t)\right)\right|_{\boldsymbol{\psi}_{j}=\boldsymbol{\psi}_{s}}+\sum_{p=1}^{l}\left[\left.\frac{\partial h_{n}\left(\boldsymbol{\psi}_{j}(t)\right)}{\partial \psi_{j p}}\right|_{\boldsymbol{\psi}_{j}=\boldsymbol{\psi}_{s}}\right]\left[\psi_{j p}(t)-\psi_{s p}(t)\right]
$$

e substituindo as expansões Eq. 15 e Eq. 16 na Eq. 7, nós temos

$$
\begin{aligned}
\frac{d \psi_{i n}(t)}{d t} & \cong f_{n}\left(\boldsymbol{\psi}_{s}(t)\right)+\sum_{p=1}^{l}\left[\left.\frac{\partial f_{n}\left(\boldsymbol{\psi}_{i}(t)\right)}{\partial \psi_{i p}}\right|_{\boldsymbol{\psi}_{i}=\boldsymbol{\psi}_{s}}\right] \delta_{i p}(t) \\
& -\varepsilon \sum_{j=1}^{N} c_{i j}\left[\left.h_{n}\left(\boldsymbol{\psi}_{j}(t)\right)\right|_{\boldsymbol{\psi}_{j}=\boldsymbol{\psi}_{s}}+\sum_{p=1}^{l}\left[\left.\frac{\partial h_{n}\left(\boldsymbol{\psi}_{j}(t)\right)}{\partial \psi_{j p}}\right|_{\boldsymbol{\psi}_{j}=\boldsymbol{\psi}_{s}}\right] \delta_{i p}(t)\right]
\end{aligned}
$$

onde substituímos na Eq. 14, assim, temos a evolução temporal para o n-ésima componente da perturbação $\delta_{i n}(t)$ que é governado por

$$
\begin{aligned}
\frac{d \delta_{i n}(t)}{d t} & \cong \sum_{p=1}^{l}\left[\left.\frac{\partial f_{n}\left(\boldsymbol{\psi}_{i}(t)\right)}{\partial \psi_{i p}}\right|_{\boldsymbol{\psi}_{i}=\boldsymbol{\psi}_{s}}\right] \delta_{i p}(t) \\
& -\varepsilon \sum_{j=1}^{N} c_{i j}\left[h_{n}\left(\boldsymbol{\psi}_{s}(t)\right)+\sum_{p=1}^{l}\left[\left.\frac{\partial h_{n}\left(\boldsymbol{\psi}_{j}(t)\right)}{\partial \psi_{j p}}\right|_{\boldsymbol{\psi}_{j}=\boldsymbol{\psi}_{s}}\right] \delta_{i p}(t)\right]
\end{aligned}
$$

Vamos assumir, por simplicidade, que a matriz de acoplamento $\mathbf{C}$ deve atender à seguinte condição

$$
\sum_{j=1}^{N} c_{i j}=0
$$

onde podemos considerar $\mathbf{C}=\boldsymbol{\Gamma}$, isto é, a matriz de acoplamento coincide com a matriz Laplaciana. Lembre-se de que a matriz Laplaciana é aquela cujos coeficientes $\gamma_{i j}$ são formados a partir das condições

$$
\gamma_{i j}=\left\{\begin{array}{c}
\kappa_{i}, \text { se } i=j \\
-1, \text { se } i \neq j \\
0, \text { outro caso, }
\end{array}\right.
$$


sendo $\kappa_{i}$ o grau de $i$-ésimo nó, obtido de

$$
\kappa_{i}=-\sum_{j=1}^{N} \gamma_{i j}
$$

A partir desta simplificação proposta (Eq. 19) podemos ver que nosso campo de interação (Eq. 6) torna-se zero quando o sistema está na variedade de sincronismo idêntico (Eq. 8), e, portanto, teríamos que

$$
\mathbf{g}\left(\boldsymbol{\psi}_{s}(t)\right)=\sum_{j=1}^{N} c_{i j} \mathbf{h}\left(\boldsymbol{\psi}_{s}(t)\right)=0
$$

pelo qual podemos excluir o termo $h_{n}\left(\boldsymbol{\psi}_{s}(t)\right)$ da Eq. 18, e assim

$$
\begin{aligned}
\frac{d \delta_{i n}(t)}{d t} & \cong \sum_{p=1}^{l}\left[\left.\frac{\partial f_{n}\left(\boldsymbol{\psi}_{i}(t)\right)}{\partial \psi_{i p}}\right|_{\boldsymbol{\psi}_{i}=\boldsymbol{\psi}_{s}}\right] \delta_{i p}(t) \\
& -\varepsilon \sum_{j=1}^{N} \nu_{i j}\left[\sum_{p=1}^{l}\left[\left.\frac{\partial h_{n}\left(\boldsymbol{\psi}_{j}(t)\right)}{\partial \psi_{j p}}\right|_{\boldsymbol{\psi}_{j}=\boldsymbol{\psi}_{s}}\right] \delta_{i p}(t)\right]
\end{aligned}
$$

onde vemos isso $c_{i j}=\gamma_{i j}=\nu_{i j}$. Como podemos ver o primeiro termo no lado direito da Eq. 23 corresponde à matriz Jacobiana da função f, qual é a dinâmica dos osciladores que compõem a rede, então nós temos

$$
\mathbf{J}_{\mathbf{f}}\left(\boldsymbol{\psi}_{s}(t)\right) \cong \sum_{p=1}^{l}\left[\left.\frac{\partial f_{n}\left(\boldsymbol{\psi}_{i}(t)\right)}{\partial \psi_{i p}}\right|_{\boldsymbol{\psi}_{i}=\boldsymbol{\psi}_{s}}\right]
$$

e por sua vez, vemos que o segundo termo no lado direito corresponde à matriz Jacobiana da função de acoplamento h, é assim que chegamos a

$$
\mathbf{J}_{\mathbf{h}}\left(\boldsymbol{\psi}_{s}(t)\right) \cong \sum_{p=1}^{l}\left[\left.\frac{\partial h_{n}\left(\boldsymbol{\psi}_{j}(t)\right)}{\partial \psi_{j p}}\right|_{\boldsymbol{\psi}_{j}=\boldsymbol{\psi}_{s}}\right]
$$

portanto, a dinâmica de cada perturbação pode ser escrita como

$$
\frac{d \delta_{i n}(t)}{d t} \cong\left[\mathbf{J}_{\mathbf{f}}\left(\boldsymbol{\psi}_{s}(t)\right)-\varepsilon \mu_{i} \mathbf{J}_{\mathbf{h}}\left(\boldsymbol{\psi}_{s}(t)\right)\right] \delta_{i n}(t)
$$

e assim vemos que o único elemento que depende do índice $i$ são os autovalores $\mu_{i}$, isso é uma vantagem, pois não devemos lidar com $N$ equações diferenciais para os estados $\boldsymbol{\psi}_{i}(t)$ que vão de $i=1,2,3 \ldots l$, senão que estudamos a dinâmica de $N$ equações diferenciais para a perturbação $\boldsymbol{\delta}_{i}(t)$, o que constitui uma simplificação do cálculo, porque o único fator que varia na Eq. 26 são os autovalores $\mu_{i}$. Tenemos entonces que la dinamica de la red de osciladores acoplados es descrita por un conjunto de $N$ blocos desacoplados onde 
sabemos que as variáveis de estado $\boldsymbol{\psi}_{i}(t)$ são projetados em cada "eigenmode" $\boldsymbol{\psi}_{i}(t)$, onde se $\boldsymbol{\psi}_{i}(t) \longrightarrow 0$ então $\boldsymbol{\delta}_{i}(t) \longrightarrow 0$. Definimos a seguinte equação

$$
\boldsymbol{P}_{i}(t)=\mathbf{J}_{\mathbf{f}}\left(\boldsymbol{\psi}_{s}(t)\right)-\varepsilon \mu_{i} \mathbf{J}_{\mathbf{h}}\left(\boldsymbol{\psi}_{s}(t)\right.
$$

que foi chamado por Pecora e Carroll em 1998 de Equação Mestra de Estabilidade (PECORA; CARROLL, 1998), portanto, podemos escrever a evolução temporal das perturbações Eq. 26 da seguinte forma

$$
\dot{\boldsymbol{\delta}}_{i}(t)=\boldsymbol{P}_{i}(t) \boldsymbol{\delta}_{i}(t)
$$

Logo, a dinâmica assintótica ao longo do $i$-ésima componente transversal $\boldsymbol{\delta}_{i}(t)$, será governado pelo maior expoente de Lyapunov $\lambda_{T}$, então $\operatorname{sim} \lambda_{T}<0$ associado a cada um das $(N-1)$ direcções tranversais, significa que as perturbações $\boldsymbol{\delta}_{i}(t) \longrightarrow 0$ para $t \longrightarrow \infty$, e a variedade de sincronismo idêntico é localmente assintoticamente estável. Caso contrário, se por algum $i$ se tem que $\lambda_{T}>0$ o sincronismo é instável. Finalmente, temos que, a dependência do maior expoente de Lyapunov $\lambda_{T}$ com os autovalores $\mu_{i}$ é o que Pecora e Carroll definiram como Função Mestra de Estabilidade. 


\section{Oscilador de Van der Pol}

Um físico holandês chamado Balthasar Van Der Pol, trabalhou na empresa Philips por meados da década de 1920, onde ele propôs um modelo matemático que permitia o estudo de certos tipos de osciladores de relaxamento devido ao interesse recente em tubos de vácuo (em especial, a lâmpada de neon) e, assim, tentar descrever os fenômenos que ocorreram naquele bulbo. O modelo matemático publicado pela primeira vez em 1926 (POL, 1926), é descrito por

$$
\ddot{y}+b\left(y^{2}-1\right) \dot{y}+y=0 .
$$

Após, vários estudos subsequentes e trabalhos relacionados com fenômenos oscilatórios (POL; MARK, 1928; KROGDAHL, 1955; FLAHERTY; HOPPENSTEADT, 1978; ROWAT; SELVERSTON, 1993; CARTWRIGHT et al., 1999; GYERGYEK; ČERČEK; STANOJEVIĆ, 1997; GARDNER et al., 2001; SPROTT, 2005; PASSOS; LOPES, 2008) foram feitos. É comum encontrar a equação de Van Der Pol quando submetida a oscilações periódicas forçadas, de modo que,

$$
\ddot{y}+b\left(y^{2}-1\right) \dot{y}+y=A \sin (\Omega t),
$$

onde Van Der Pol observou particularmente o surgimento do caos em osciladores de relaxamento com uma lâmpada de neon e assim a Eq. 30 passou a modelar seu comportamento razoavelmente bem.

Vamos ver que podemos escrever o sistema Van Der Pol bidimensional sem forçamento externo da seguinte maneira

$$
\left\{\begin{array}{l}
\dot{x}=-y-b\left(1-y^{2}\right) x, \\
\dot{y}=x
\end{array}\right.
$$

o que nos permite integrar numericamente as equações de estado e capturar toda a natureza dinâmica do referido sistema.

\subsection{Modelo Macroeconômico}

Os sistemas econômicos são exemplos de sistemas complexos e sua imprevisibilidade deve-se a não linearidade, à semelhança do que acontece nos sistemas caóticos. Como é 
sabido, ainda existe controvérsia sobre a presença de caos em dados econômicos devido ao tamanho das séries empíricas e à suposta alta dimensionalidade do sistema (KYRTSOU; VORLOW, 2005). Entretanto, as economias apresentam comportamentos coletivos como mudanças estruturais (bifurcações) que podem ser estudados por meio da modelagem matemática (BARNETT; DUZHAK, 2010; VOLOS; KYPRIANIDIS; STOUBOULOS, 2012). O aspecto interessante é que os padrões observados em sistemas ecológicos ou físicos também aparecem em outros sistemas tais como econômicos e fisiológicos. Por exemplo, oscilações e sincronizações de fase são comportamentos dinâmicos estudados na Física, cujos padrões também aparecem em populações de vaga-lumes, em séries de batimentos cardíacos ou nos ciclos circadianos em humanos (MONTEIRO, 2010). Assim, não é surpresa que os modelos paradigmáticos para o estudo das oscilações na Física possam ser adaptados para estudar ciclos econômicos. Algumas variáveis econômicas agregadas exibem um tipo de flutuação chamada de ciclos de negócios. Estes ciclos consistem em expansões que ocorrem quase ao mesmo tempo em muitas atividades econômicas, seguidas de recessões, contrações e recuperação (CALDERóN; FUENTES, 2014). Essa sequência de mudanças é recorrente, mas não apresenta duração e períodos bem comportados. Entre as variáveis, podemos citar consumo, investimento, produto interno bruto e estoque de capital, que seguem um movimento oscilatório incorporando um vasto espectro de ciclos. Esses comportamentos oscilatórios tem causa endógena e podem ser descritos por meio de equações diferenciais não lineares (GOODWIN, 1967; VOLOS; KYPRIANIDIS; STOUBOULOS, 2012).

Bouali et al. (BOUALI et al., 2012) propuseram uma modificação do oscilador de Van de Pol bidimensional (Eq. 31) que fosse capaz de representar um sistema macroecônomico idealizado. Para isto, eles introduziram uma terceira equação e fizeram a interpretação das variáveis de estado no contexto macroeconômico. O modelo é descrito por três equações diferenciais ordinárias, a saber:

$$
\left\{\begin{array}{l}
\dot{x}=m \cdot y+p \cdot x \cdot\left(d-y^{2}\right), \\
\dot{y}=-x+c \cdot z \\
\dot{z}=s \cdot x-r \cdot y
\end{array}\right.
$$

As variáveis de estado $x, y$ e $z$ representam a poupança das famílias, o produto interno bruto (PIB) e a entrada ou fluxo de capital respectivamente. Os parâmetros $m, p, d, c, s$ e $r$ são positivos e representam respectivamente os índices: propensão marginal a consumir, fator de lucro capitalizado do investimento, potencial do PIB, fator de saida do capital, 
fator de propensão a poupar e o fator do endividamento. Este sistema possui três pontos de equilíbrio:

$$
\left\{\begin{array}{l}
P_{1}=(0,0,0) \\
P_{2}=(\alpha, s \alpha / r,-\alpha / c), \\
P_{3}=-E_{2} .
\end{array}\right.
$$

com

$$
\alpha=\frac{p d+m\left(\frac{s}{r}\right)}{\left(\frac{s}{r}\right)^{2} p}
$$

Para os parâmetros

$$
\begin{cases}m=0.02, & p=0.4, \\ d=1.0, & c=50, \\ s=10, & r=0.1,\end{cases}
$$

o sistema exibe comportamento caótico conforme apresentado por Bouali utilizando-se do algoritmo de Wolf et al. (WOLF et al., 1985), ilustrado na Fig. 4.

O sistema descrito pelo conjunto de equações Eq. 32 exibe uma riqueza de comportamentos dinâmicos quando são variados os parâmetros de controle conforme discutido por Bouali (BOUALI, 1999). Vale ressaltar que estas economias podem ser abertas e realizar trocas com outras economias sujeitas a flutuações. Como as economias interagentes podem estar em fases diferentes dos ciclos de negócios, é interessante estudar o que acontece com várias economias acopladas. Portanto, na próxima sessão, estudaremos uma rede de economias acopladas cuja dinâmica individual é modelada pelo conjunto de equações que observamos.

\subsection{Sistema Macroeconômico Acoplado}

Recentemente, Volos et al. (VOLOS; KYPRIANIDIS; STOUBOULOS, 2012) estudaram a interação de duas economias. Para isto, eles acoplaram dois modelos de Bouali na variável que descreve o fluxo de entrada de capital do exterior, obtendo assim as seguintes equações

$$
\text { Economia } 1=\left\{\begin{array}{l}
\dot{x}_{1}=m y_{1}+p x_{1}\left(d-y_{1}^{2}\right), \\
\dot{y}_{1}=-x_{1}+c z_{1}, \\
\dot{z}_{1}=s x_{1}-r y_{1}+\xi_{1}\left(z_{2}-z_{1}\right),
\end{array}\right.
$$




$$
\text { Economia } 2=\left\{\begin{array}{l}
\dot{x}_{2}=m y_{2}+p x_{2}\left(d-y_{2}^{2}\right), \\
\dot{y}_{2}=-x_{2}+c z_{2}, \\
\dot{z}_{2}=s x_{2}-r y_{2}+\xi_{2}\left(z_{1}-z_{2}\right),
\end{array}\right.
$$

Propusemos uma generalização para $N$ economias acopladas, seguindo tal modelo de acoplamento difusivo proposto. O conjunto de $N$ economias acopladas em forma de anel é dado pelas seguintes equações diferencias:

$$
\left\{\begin{array}{l}
\dot{x}_{i}=m y_{i}+p x_{i}\left(d-y_{i}^{2}\right) \\
\dot{y}_{i}=-x_{i}+c z_{i} \\
\dot{z}_{i}=s x_{i}-r y_{i}+\xi_{i}\left(z_{i+1}-z_{i}\right)
\end{array}\right.
$$

com $i=1,2, \ldots, N$. A variação $z_{i+1}-z_{i}$ representa o saldo na balança comercial do país $i$ com o país $i+1$. A balança comercial pode ser utilizada para financiar os déficits orçamentários do governo ou pode ser usado para aumentar a produção. Assim, $\xi_{i}$ mede o efeito da balança comercial sobre a economia.

Considerando cada economia como um sistemas dinâmico complexo, pode-se usar inúmeras técnicas matemáticas para caracterizar os padrões que elas exibem ao variarmos os parâmetros relevantes, bem como estudar a estabilidade das soluções. Além disso, do ponto de vista econômico, o sistema simula a fuga de capitais observada nos países menos desenvolvidos. Então, aqui examinamos o efeito da entrada e saída de capital entre dois sistemas econômicos conjugados. O estudo da estabilidade por meio da função mestra de estabilidade (PECORA; CARROLL, 1998; HUANG et al., 2009; MONTEIRO, 2010), será apresentado posteriormente.

\subsection{Comportamentos Dinâmicos}

\subsubsection{Atrator do Sistema}

Uma das primeiras etapas no estudo da dinâmica caótica do sistema é observar as relações das variáveis de estado no espaço de fase. Vamos ver na Fig. 4 que existe uma relação funcional não trivial entre as variáveis de estado $x(t), y(t)$ e $z(t)$, caracterizadas pela existência de um atrator estranho bem definido para a dinâmica do sistema de Van 
Der Pol isolado (Eq. 32). Por sua vez, observamos as projeções $x y$ e $x z$ para os seguintes parâmetros: $(m, p, d, c, s, r)=(0.02,0.4,1.0,50,10,0.1)$ respectivamente.

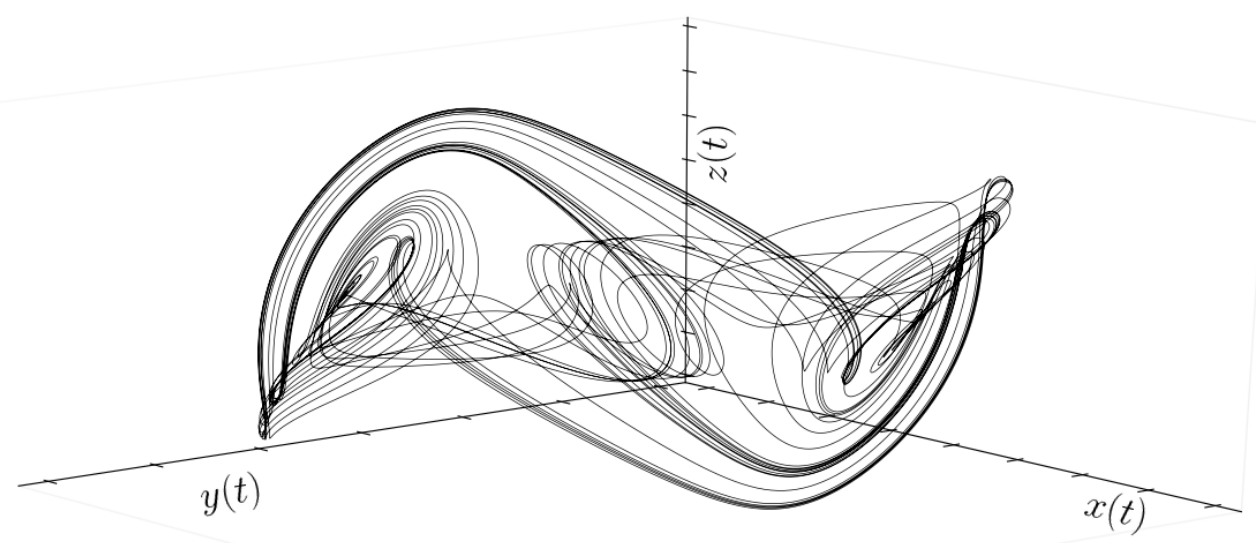

Figura 4 - Atrator tridimensional das variáveis $x, y, z$, associado ao sistema Eq. 32 para os seguintes parâmetros: $(m, p, d, c, s, r)=(0.02,0.4,1.0,50,10,0.1)$ respectivamente. Fonte: Elaborado pelo autor.
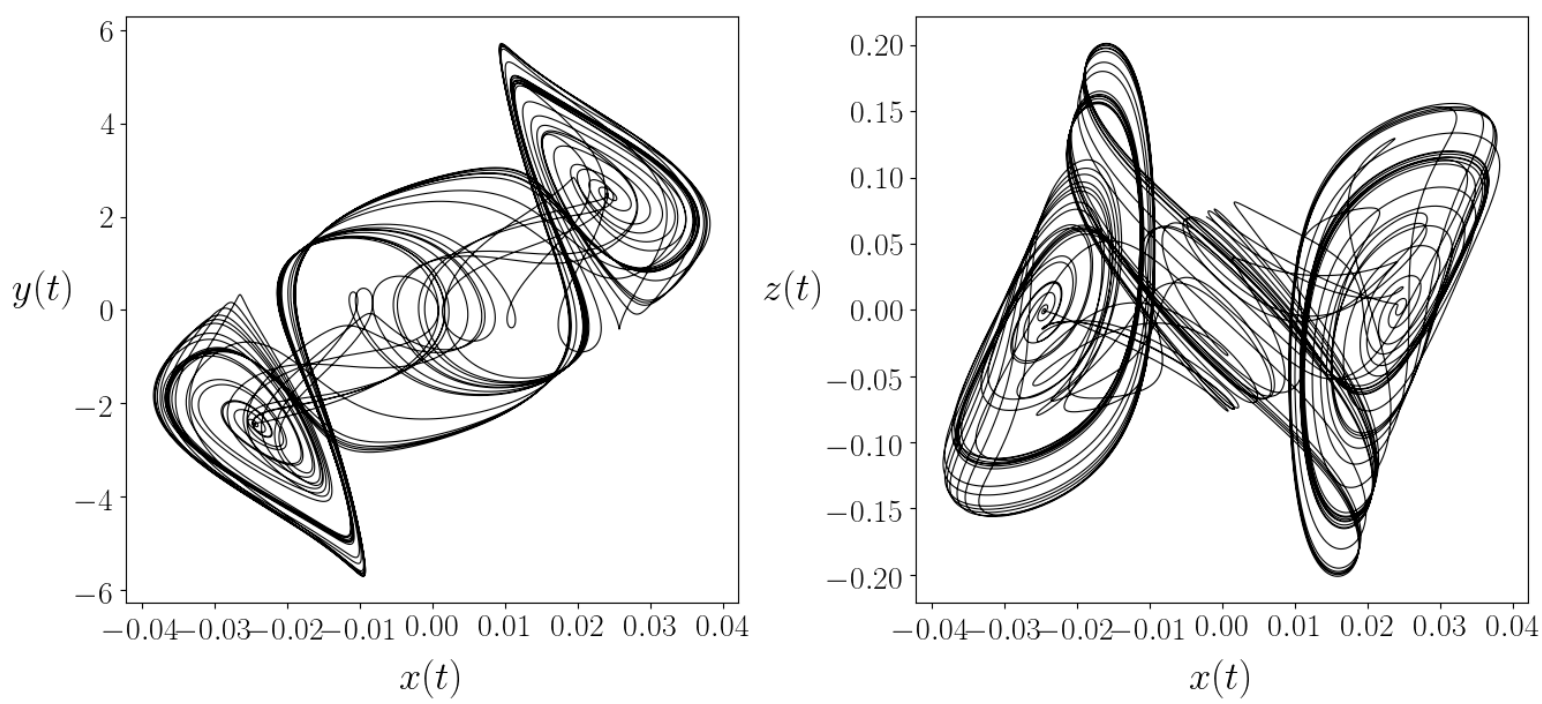

Figura 5 - Projeções $x y$ e $x z$ do atrator tridimensional associado ao sistema Eq. 32 para os seguintes parâmetros: $(m, p, d, c, s, r)=(0.02,0.4,1.0,50,10,0.1)$ respectivamente. Fonte: Elaborado pelo autor. 
Podemos analisar o sistema isolado Eq. 32 efetuando um "feedback" negativo, portanto, escreveremos as equações da seguinte forma

$$
\left\{\begin{array}{l}
\dot{x}=m y+p x\left(d-y^{2}\right), \\
\dot{y}=-x+c z \\
\dot{z}=s x-r y-\xi z
\end{array}\right.
$$

assim podemos ver as mudanças na dinâmica do atrator do sistema para um oscilador com feedback sobre sua variável de estado $z$ (Fig. 6). Para $\xi=0$ obtemos o atrator observado anteriormente na projeção $z x$ (Fig. 6(a)), e para valores acima de $\xi=1.0$, o sistema mostra um ciclo limite (Fig. 6(b)), simulando assim os ciclos econômicos esperados.
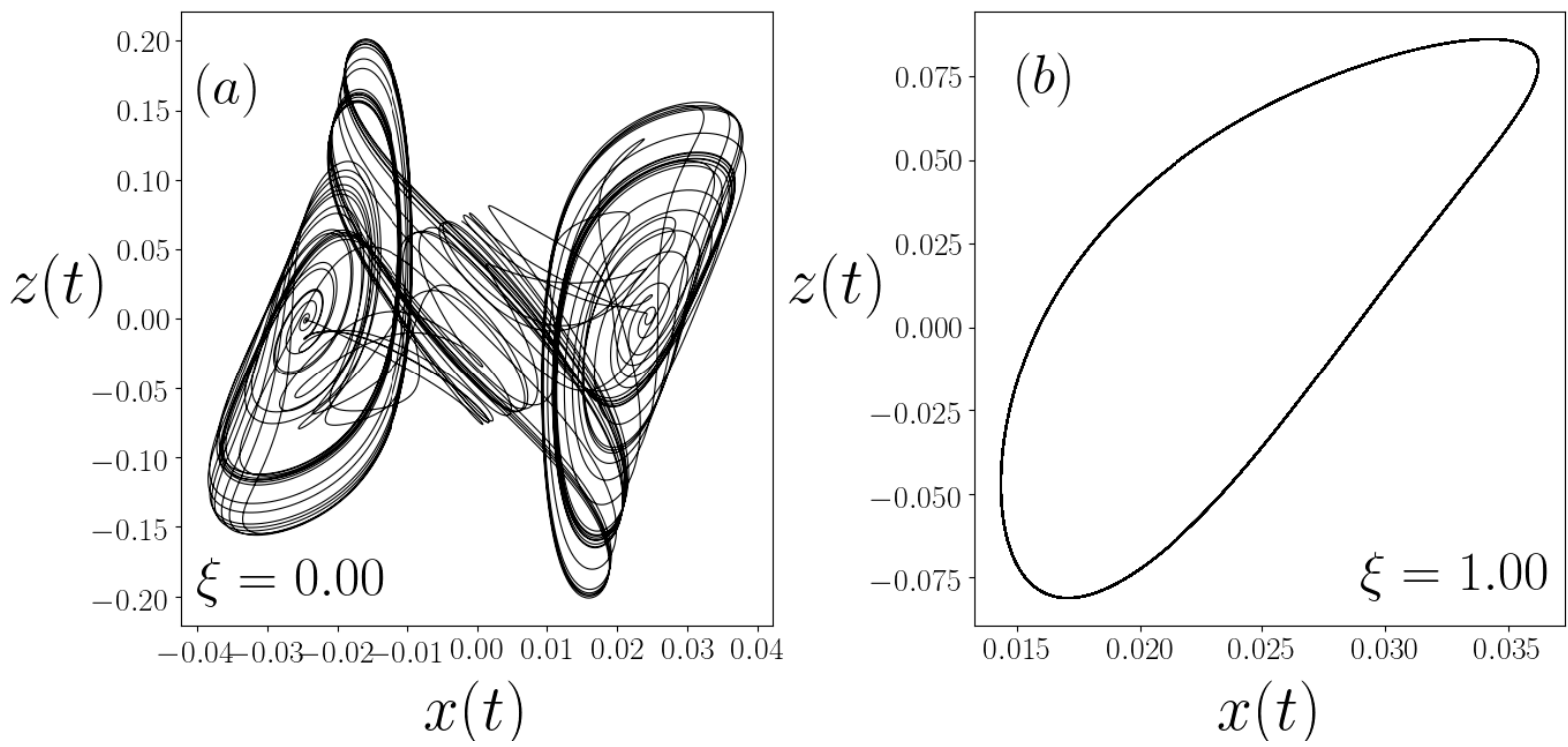

Figura 6 - Projeções $z x$ dos atratores do sistema Eq. (39) para diferentes "feedbacks"; (a) $\xi=0.0$ e $(b) \xi=1.0$; com os seguintes parâmetros: $(m, p, d, c, s, r)=$ $(0.02,0.4,1.0,50,10,0.1)$ respectivamente. Os cálculos foram feitos com $5 \times 10^{4}$ iterações, ignorando $4 \times 10^{4}$ transientes e, portanto, considerando $1 \times 10^{4}$ iterados após os transientes. Fonte: Elaborado pelo autor.

Observamos na Fig. 7 que o atrator muda para dois conjuntos diferentes de condições iniciais, positivas e negativas. Primeiro, vemos que quando o sistema está isolado, isso é $\xi=0.0$, tem órbitas semelhantes para ambas as condições iniciais positivas $a) x(0), y(0), z(0)=0.05,0.20,0.02$, quanto às condições iniciais negativas $b$ ) $x(0), y(0), z(0)=-0.05,-0.20,-0.02$. Quando ativamos o termo de feedback negativo sobre a variável $z$, observamos que o sistema tende a um ciclo limite, como vimos na Fig. 7. 


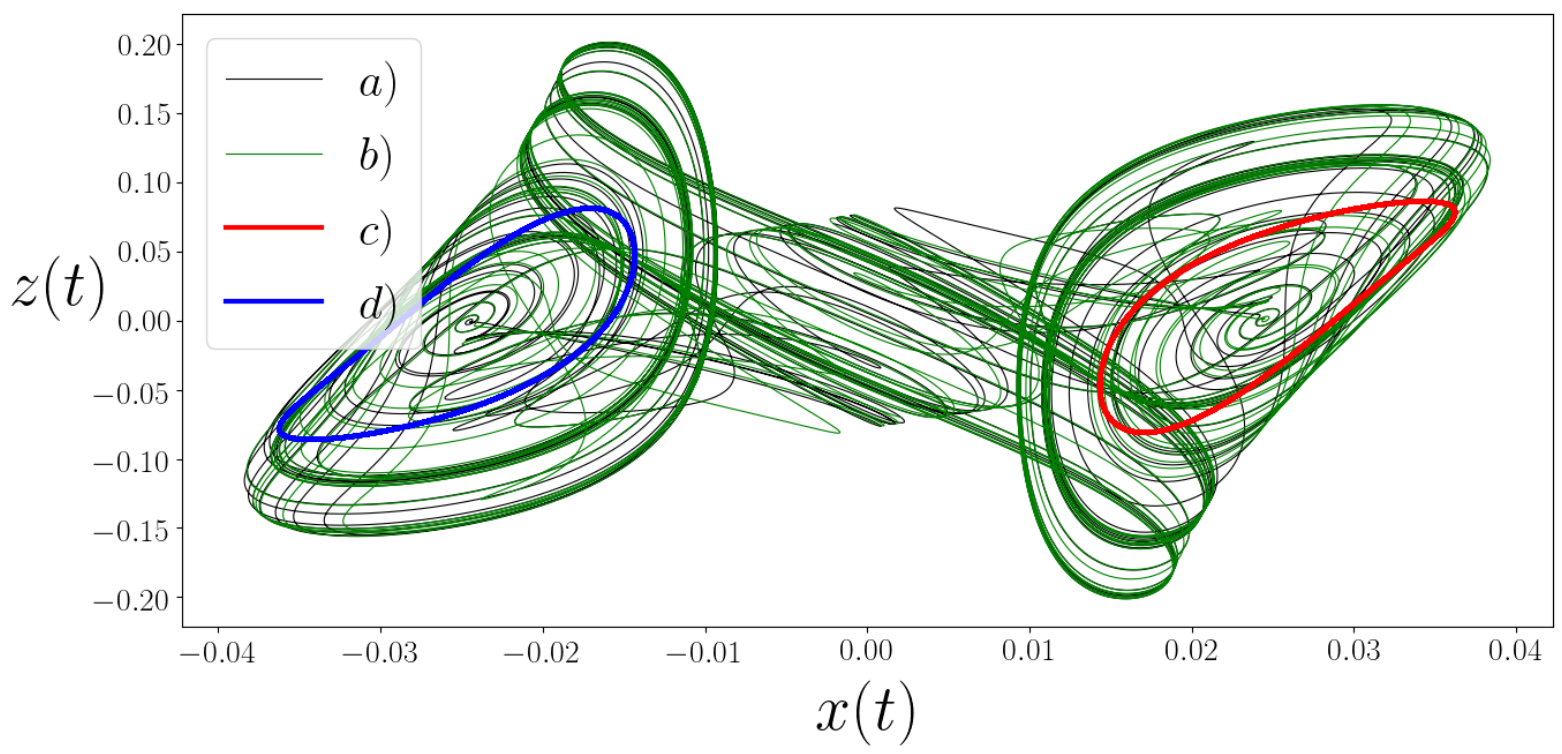

Figura 7 - Atrator do sistema Eq. 39 para diferentes "feedbacks", e com condições iniciais positivas $x(0), y(0), z(0)=0.05,0.20,0.02$ e negativas $x(0), y(0), z(0)=$ $-0.05,-0.20,-0.02$; temos que a) $\xi=0.0$ com condições iniciais positivas, b) $\xi=0.0$ com condições iniciais negativas, $c) \xi=1.0$ com condições iniciais positivas e $d$ ) $\xi=1.0$ com condições iniciais negativas; com os seguintes parâmetros: $(m, p, d, c, s, r)=(0.02,0.4,1.0,50,10,0.1)$ respectivamente.

É interessante notar que as diferentes condições iniciais testadas levaram a diferentes atratores de ciclo-limite, no lóbulo à direita e outro no lóbulo esquerdo do atrator estranho quando $\xi=0$ (Fig. 7). Isto indica a existência de biestabilidade ou multiestabilidade do sistema devido ao aumento do parâmetro de feedback.

\subsubsection{Expoentes de Lyapunov e Dimensão Fractal}

Até agora, nós fomos capazes de estudar a dinâmica natural do nosso sistema (Eq. 32) através da observação da relação de suas variáveis de estado no espaço de fase, que descrevem um atrator externo 4. Vimos também como é a evolução temporal da dinâmica de uma rede composta por vários desses osciladores Eq. 38 através dos padrões espaço temporais que eles descrevem, o que nos dá um certo grau de confiança quando se trata de falar sobre um sistema caótico.

A partir da análise realizada no capítulo 2, Sabemos que uma maneira de abordar uma análise mais objetiva do comportamento do nosso sistema com feedback é através do estudo de expoentes de Lyapunov (LYAPUNOV, 1893; LYAPUNOV; WALKER, 1994), 
que constituem uma ferramenta adequada para analisar a evolução de duas trajetórias infinitesimalmente próximas e, assim, observar a sensibilidade às condições iniciais, e ser capaz de confirmar a existência do caos.

O parâmetro de controle $c$ que representa o fator de saída de capital, constitui o parâmetro de acoplamento da variável de fluxo de capital na macroeconomia modelada, e, assim, gera a tridimensionalidade do sistema original Van Der Pol. Portanto, é importante observar o comportamento do sistema com feedback proposto (Eq. 39) variando esse fator de saída. Portanto, calculamos os expoentes de Lyapunov $\lambda$ em função de $c$ para nosso sistema Eq. 39 com uma intensidade de feedback de $\xi=1.0$, como mostra a Fig. 8, onde observamos claramente o espectro dos três maiores expoentes de Lyapunov $\lambda_{1}, \lambda_{2}$ e $\lambda_{3}$ correspondente a cada uma das variáveis de estado do sistema. Vemos claramente como o sistema começa a ter um comportamento de ponto fixo até um valor de $\xi=32$ onde passa a ter um comportamento de ciclo limite para valores maiores de $c$.

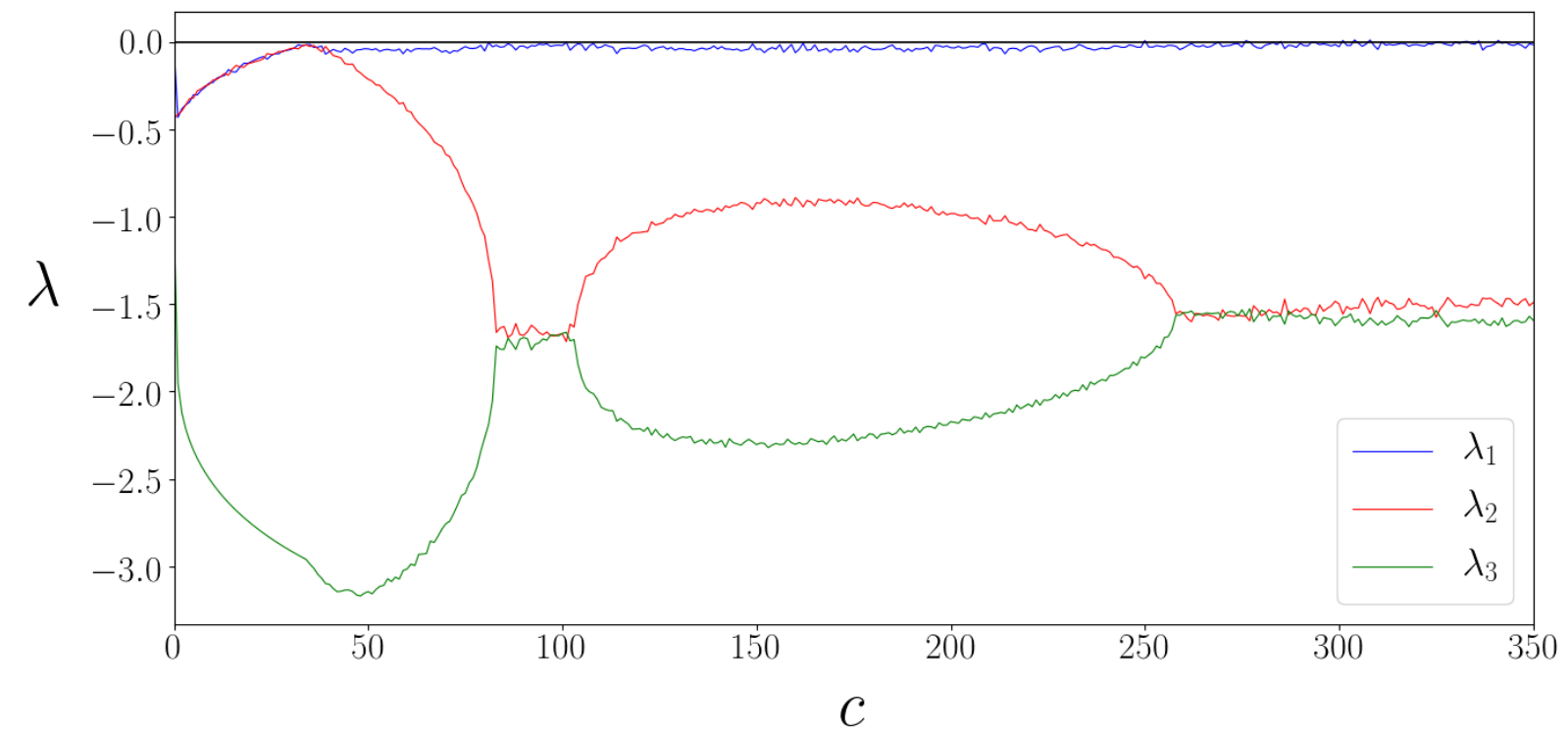

Figura 8 - Espectro dos Maiores Expoentes de Lyapunov $\lambda_{1}, \lambda_{2}$ e $\lambda_{3}$ em função do parâmetro de controle $c$ para o sistema Eq. (39) com uma intensidade de feedback de $\xi=1.0$ e com $(m, p, d, c, s, r)=(0.02,0.4,1.0,50,10,0.1)$. Fonte: Elaborado pelo autor.

Consequentemente, podemos agora através da conjectura de Kaplan-Yorke (KAPLAN; YORKE, 1979; FREDERICKSON et al., 1983) calcular a dimensão fractal do 
nosso sistema com feedback (Eq. 39), pois é possível organizar cada um dos $k$ Expoentes de Lyapunov de tal maneira que

$$
\lambda_{1} \geq \lambda_{2} \geq \cdots \geq \lambda_{k}
$$

onde,

$$
\sum_{i=1}^{k} \lambda_{i} \geqslant 0
$$

assim também,

$$
\sum_{i=1}^{k+1} \lambda_{i}<0,
$$

portanto, a conjectura de Kaplan-Yorke afirma que a dimensão fractal do sistema é dada por

$$
D_{\mathrm{KY}}=k+\sum_{i=1}^{k} \frac{\lambda_{i}}{\left|\lambda_{k+1}\right|} .
$$

Agora podemos calcular simultaneamente $D_{K Y}$ em função do parâmetro $c$ e, assim, observar como o fluxo de capital altera a dimensionalidade da macroeconomia. Podemos ver como a dimensão fractal é $D_{K Y}=0$ para valores menores que $\xi=32$ e $D_{K Y}=1$ para valores mais altos, corroborando assim os ciclos econômicos gerados por essa dinâmica de feedback.

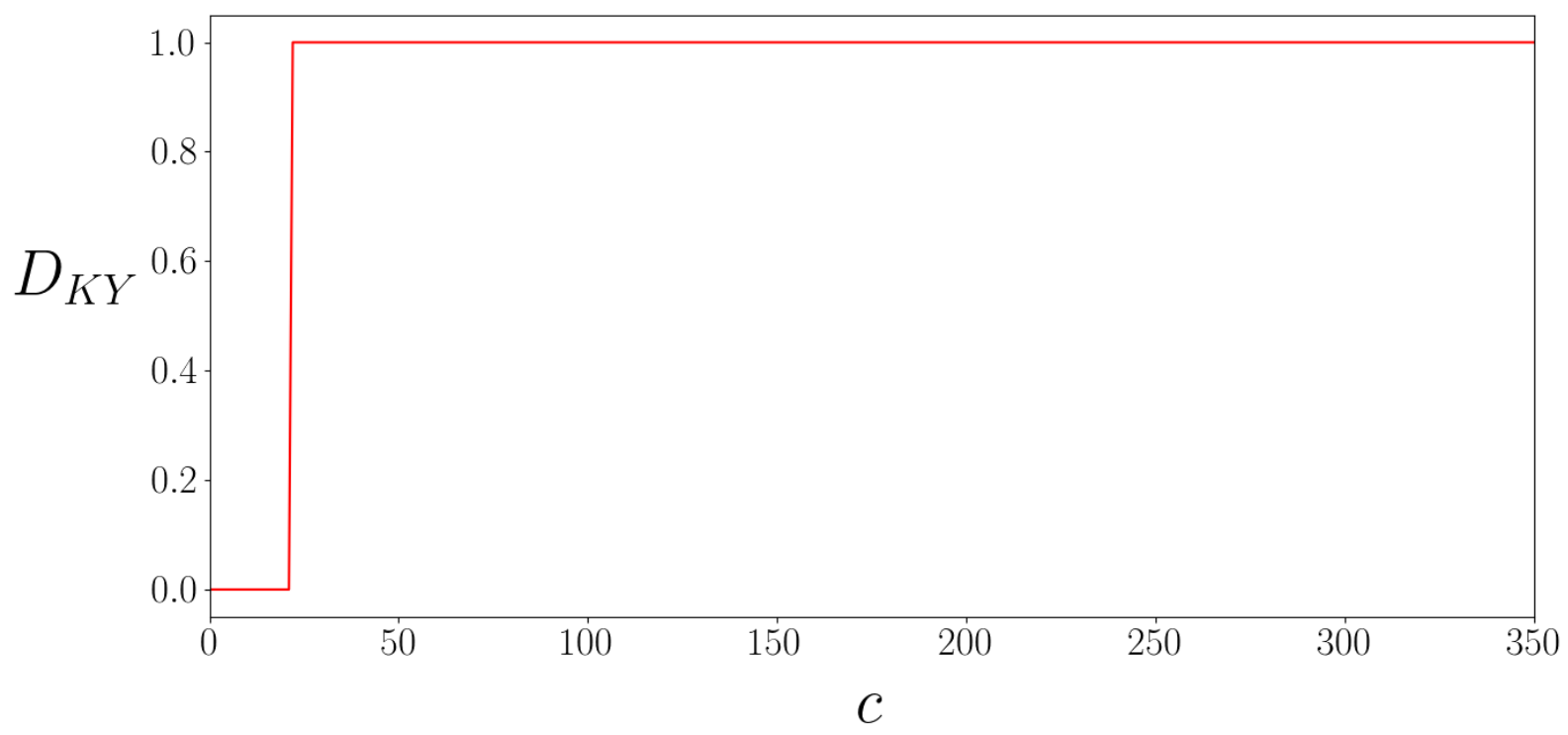

Figura 9 - Dimensão Kaplan-Yorke como função do parâmetro de controle $c$ para o sistema Eq. 39 com uma intensidade de feedback de $\xi=1.0$ e com $(m, p, d, c, s, r)=$ $(0.02,0.4,1.0,50,10,0.1)$. Fonte: Elaborado pelo autor. 
Ao mesmo tempo, é importante ver como a intensidade do parâmetro de acoplamento $\xi$ para nosso sistema macroeconommico com feedback Eq. 39, influencia a dinâmica caótica do sistema, e, assim, ser capaz de observar o espectro dos maiores expoentes de Lyapunov. Podemos observar na Fig. 10, o espectro dos três maiores Expoentes de Lyapunov $\lambda_{1}, \lambda_{2}$ e $\lambda_{3}$ em função de $\xi$.

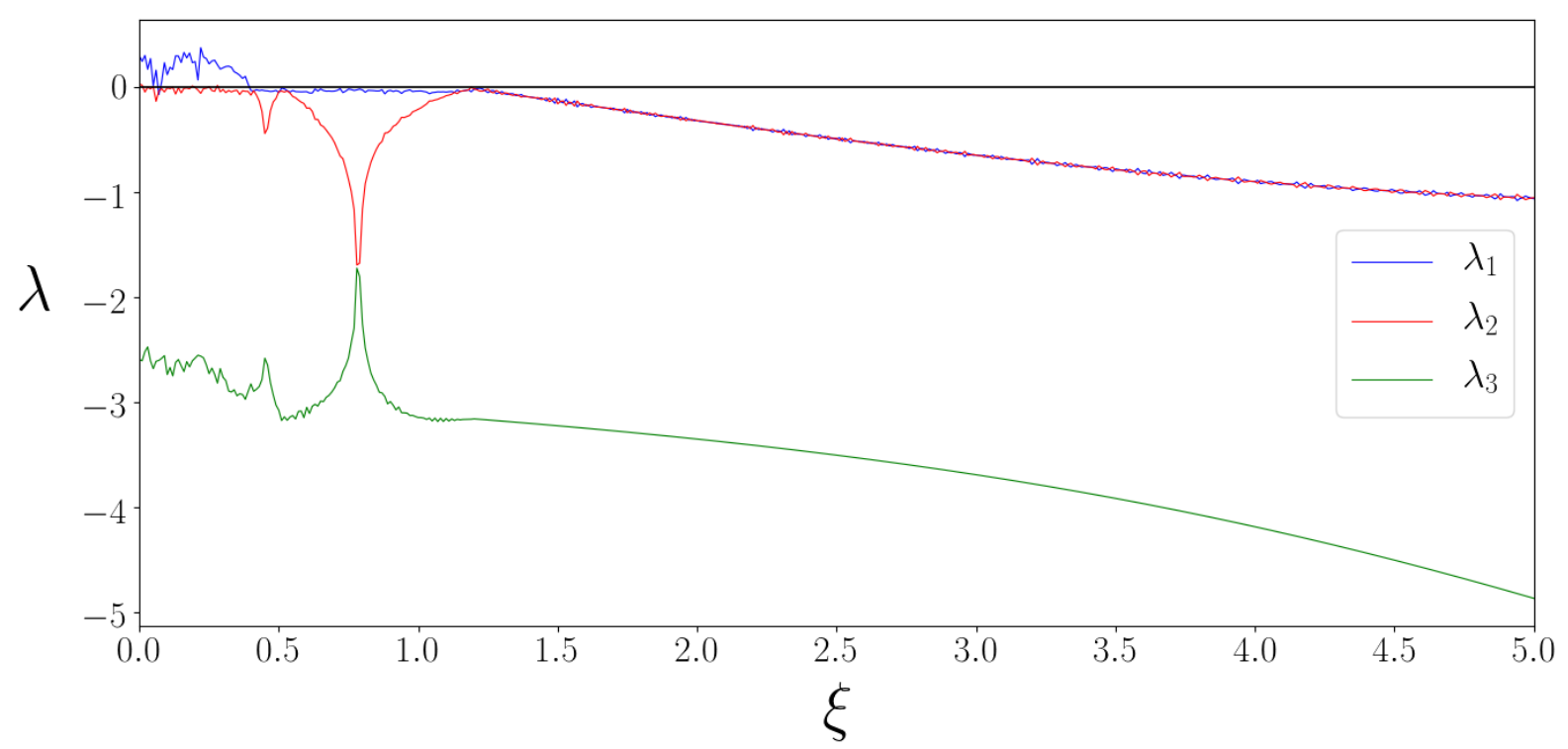

Figura 10 - Espectro dos Maiores Expoentes de Lyapunov $\lambda_{1}, \lambda_{2}$ e $\lambda_{3}$ em função do parâmetro de acoplamento $\xi$ para o sistema Eq. 39; $\operatorname{com}(m, p, d, c, s, r)=$ $(0.02,0.4,1.0,50,10,0.1)$. Fonte: Elaborado pelo autor.

É interessante observar como o sistema tem um comportamento caótico para valores pequenos do parametro de acoplamento $\xi$ e comporta-se como um ciclo limite entre os valores $0.4 \lesssim \xi \lesssim 1.33$ para logo se tornar um ponto fixo para valores maiores. Assim, graças ao espectro dos maiores expoentes de Lyapunov calculado, podemos determinar a dimensionalidade do sistema em função do parâmetro de acoplamento $\xi$, e podemos ver na Fig. 11 como a dimensão fractal varia. 


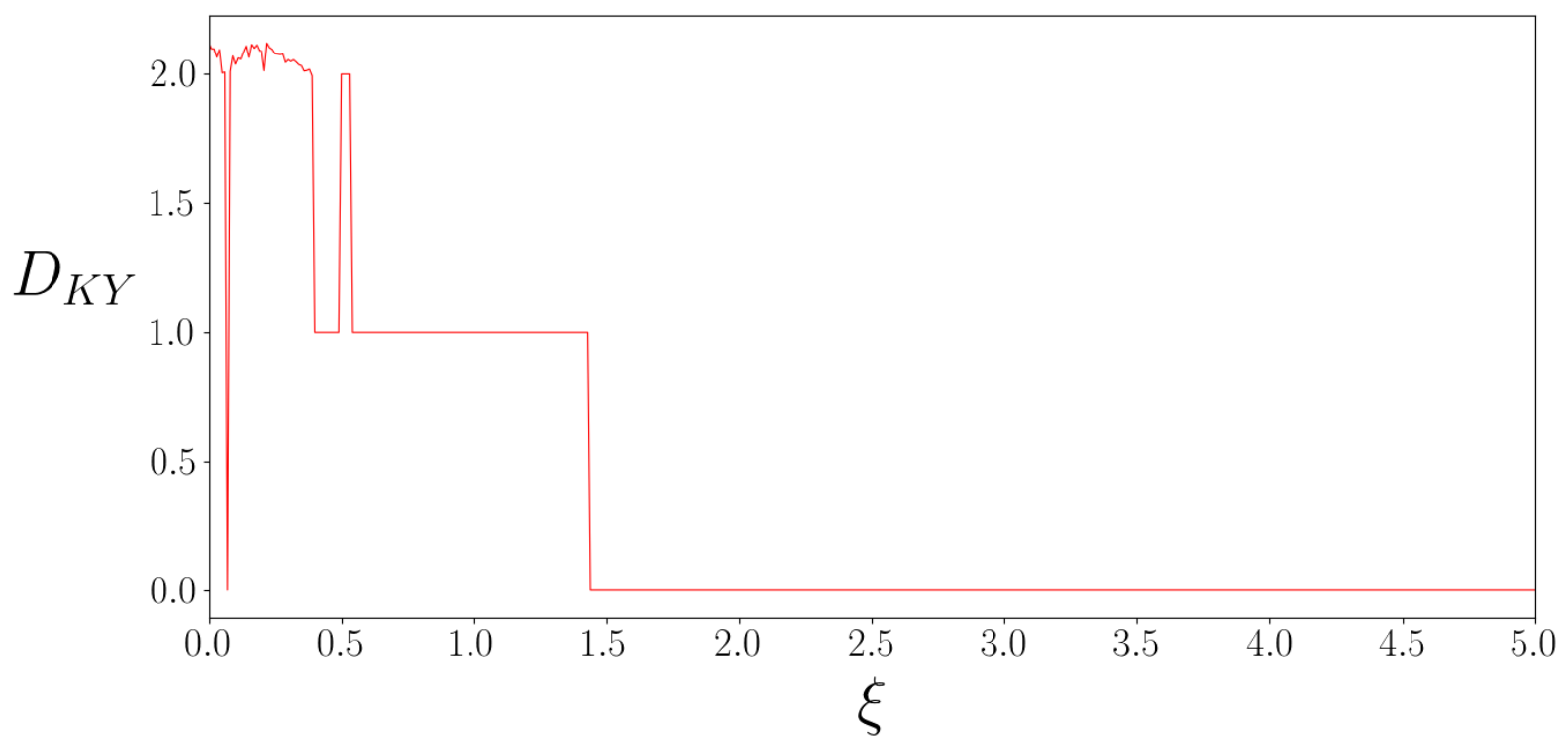

Figura 11 - Dimensão Kaplan-Yorke como função do parâmetro de acoplamento $\xi$ para o sistema Eq. 39; com $(m, p, d, c, s, r)=(0.02,0.4,1.0,50,10,0.1)$. Fonte: Elaborado pelo autor.

Observamos que podemos corroborar as mudanças no atrator do sistema, que passam de ter uma dimensão fractal para se comportar como um ciclo limite e depois convergir para um ponto fixo. 


\section{Sincronização}

Foi demonstrado que, para dois ou mais sistemas caóticos acoplados com oscilações periódicas irregulares, existem estados de sincronização em que cada uma das variáveis de estado ajusta sua dinâmica para que sejam iguais em cada um dos sistemas, tudo isso devido à influência do parâmetro de acoplamento que permite a interação e troca de sinais entre os referidos sistemas (PIKOVSKY et al., 2003). Lembre-se de que um dos primeiros resultados relatados nos quais a existência de sincronização em sistemas que exibem oscilações periódicas irregulares foi descrita por Huygens no ano de 1665, onde observo o movimento de dois relógios pendulares fracamente acoplados por meio de um barra de madeira (HUYGENS; OSCILLATORIUM, 1986). A importância de estudar o fenômeno da sincronização é que ele existe com grande frequência na natureza e é um processo através do qual sistemas como biológicos, econômicos, sociais, epidemiológicos, meteorológicos etc. exibem comportamentos particulares que nos permitem abordar para resolver o problema em estudo. O acoplamento bidirecional entre as variáveis de estado do sistema Eq. 38, permite a interação mútua entre cada um dos osciladores que compõem o sistema, como vimos no diagrama da Fig. 3, porque o principal objetivo desse tipo de acoplamento é que as órbitas converjam para uma variedade invariável. Esse tipo de acoplamento bidirecional foi encontrado em alguns sistemas ópticos, biológicos e também em comportamentos neuronais (SHAHVERDIEV; SHORE, 2009; LIU et al., 2012).

Geralmente, se tivermos dois ou mais sistemas idênticos acoplados, uma das formas mais simples de sincronização que podemos observar é a chamada sincronização completa, onde todas as trajetórias de cada uma das variáveis do sistema são iguais entre si. Agora, o oposto se aplica da mesma forma, que é quando todos os estados estão completamente fora de sincronia, chegando a ter um estado de dessincronização completa. Existem outros tipos de estados sincronizados no meio dos limites de sincronização completa e dessincronização completa, esses estados são o surgimento de sincronização generalizada, sincronização de fase, sincronização Lag, sincronização inversa, sincronização proyectiva y sincronização impulsiva. 


\subsection{Caracterização de Estados Sincronizados}

Como estudamos no capítulo 2, quando todas as variáveis de estado estiverem no variedade de sincronismo idêntico podemos dizer que a sincronização no sistema Eq.38 corresponde, para um certo tempo $t$, a um estado em que

$$
\boldsymbol{\psi}_{i}(t)=\boldsymbol{\psi}_{j}(t)=\boldsymbol{\psi}_{s}(t) \quad \forall i, j
$$

onde $\boldsymbol{\psi}_{s}(t)$ é a solução para a qual o sistema Eq. 38 converge na variedade de sincronismo idêntico. Esta condição de sincronização pode ser descrita da seguinte maneira

$$
\boldsymbol{\psi}_{i}(t)=\overline{\boldsymbol{\psi}}(t) \forall i
$$

onde $\overline{\boldsymbol{\psi}}(t)$ é o campo medio instantâneo do sistema em estudo, que é dado por

$$
\overline{\boldsymbol{\psi}}(t)=\frac{1}{N} \sum_{i=1}^{N} \boldsymbol{\psi}_{i}(t)
$$

que corresponde à soma de cada uma das variáveis de estado de cada um dos osciladores que compõem a rede em questão.

Para caracterizar todos os estados possíveis de sincronização do sistema Eq. 38, consideraremos o desvio padrão instantâneo $\sigma_{t}$ da distribuição de variáveis de estado $\boldsymbol{\psi}_{i}(t)$, que é dado por

$$
\sigma_{t}=\left[\frac{1}{N} \sum_{i=1}^{N}\left(\boldsymbol{\psi}_{i}(t)-\overline{\boldsymbol{\psi}}(t)^{2}\right]^{1 / 2},\right.
$$

onde depois de descartar um certo número de tempo transitório $T$, calculamos o desvio padrão assintótico $\langle\sigma\rangle$, definido da seguinte forma

$$
\langle\sigma\rangle=\frac{1}{T} \sum_{t=1}^{T} \sigma_{t}
$$

assim, diremos que um estado sincronizado corresponde a

$$
\langle\sigma\rangle=0
$$

onde, para as simulações numéricas realizadas, consideramos o critério numérico $\langle\sigma\rangle<10^{-7}$ para caracterizar os valores zero desta medida. 


\subsection{Estados de Sincronização}

Tendo descrito uma ferramenta para estudar o surgimento da sincronização, passamos a estudar os estados de sincronização da rede de osciladores de Van Der Pol proposta Eq. 38, onde para isso vamos considerar as variáveis $x_{i}, y_{i}, z_{i}$ para esse cálculo, portanto, temos que

$$
\sigma_{t}=\left[\frac{1}{N} \sum_{i=1}^{N}\left(x_{i}-\bar{x}\right)^{2}+\left(y_{i}-\bar{y}\right)^{2}+\left(z_{i}-\bar{z}\right)^{2}\right]^{1 / 2} .
$$

A seguir, estamos interessados na evolução do sistema quando o parâmetro de acoplamento é aumentado. Lembre-se de que a variável de estado $y(t)$ representa o PIB do modelo macroeconômico, portanto, estamos interessados em saber quando essas variáveis de estado entram em coerência ou sincronização. Do ponto de vista geral, são possíveis dois tipos de acoplamento, que permitem ao sistema alcançar estados de sincronização, chamados de acoplamento unidirecional e bidirecional. Vamos estudar o comportamento do sistema macroeconômico modelado Eq. 38 sujeito a esta primeira configuração de acoplamento. O primeiro tipo de acoplamento chamado de acoplamento unidirecional, caracteriza-se por ter uma configuração "mestre-escravo", em que um dos subsistemas exerce influência sobre outro subsistema sem receber resposta deste, vamos observar esse tipo de acoplamento para uma rede de osciladores $N=2$

$$
\begin{gathered}
\text { Mestre }=\left\{\begin{array}{l}
\dot{x}_{1}=m y_{1}+p x_{1}\left(d-y_{1}^{2}\right), \\
\dot{y}_{1}=-x_{1}+c z_{1}, \\
\dot{z}_{1}=s x_{1}-r y_{1},
\end{array}\right. \\
\text { Escravo }=\left\{\begin{array}{l}
\dot{x}_{2}=m y_{2}+p x_{2}\left(d-y_{2}^{2}\right), \\
\dot{y}_{2}=-x_{2}+c z_{2}, \\
\dot{z}_{2}=s x_{2}-r y_{2}+\xi\left(z_{1}-z_{2}\right),
\end{array}\right.
\end{gathered}
$$

neste caso, o parâmetro de acoplamento $\xi_{1}=0$, y $\xi_{2}=\xi \geq 0$, portanto, temos influência unidirecional do sistema mestre sobre o escravo. Podemos observar na Fig. 12 para uma rede de $N=2$ osciladores acoplados e com o arranjo de parâmetros fixos $(m, p, d, c, s, r)=$ $(0.02,0.4,1.0,50,10,0.1)$ respectivamente, os estados de sincronização para diferentes condições iniciais em cada iteração temporal. Observamos que o sistema entra rapidamente 
em estados completos de sincronização para um valor de parâmetro de acoplamento $\xi=0.18$.

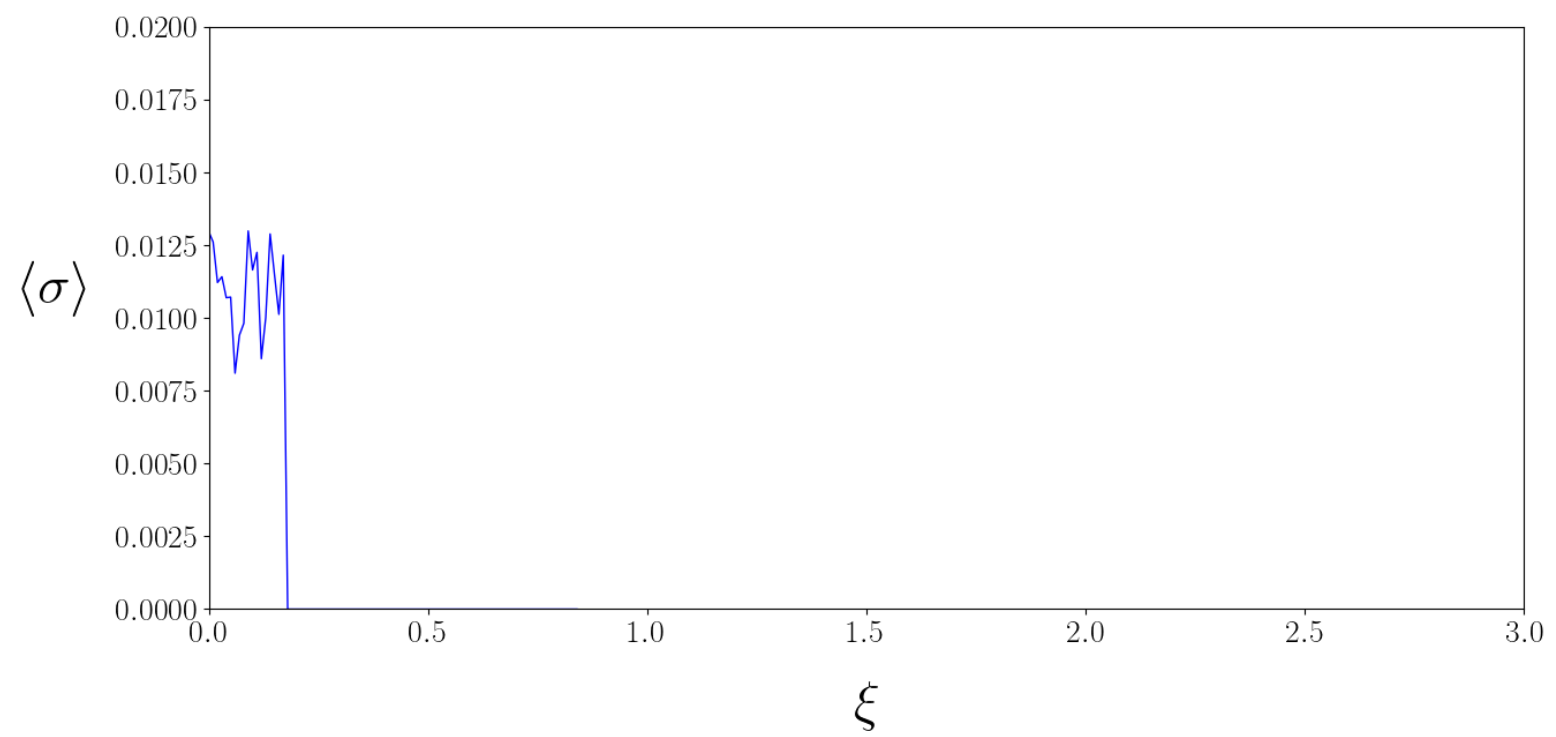

Figura 12 - Diagrama de sincronização do sistema Eq. (38) para uma rede de $N=2$ nós em função do parâmetro de acoplamento $\xi$ associado aos estados $x_{i}, y_{i}, z_{i}$ para diferentes condições iniciais em cada iteração temporal; $\operatorname{com}(m, p, d, c, s, r)=$ $(0.02,0.4,1.0,50,10,0.1)$; acoplamento do tipo unidirecional. Os cálculos foram feitos com $5 \times 10^{4}$ iterações, ignorando $4 \times 10^{4}$ transientes e, portanto, considerando $1 \times 10^{4}$ iterados após os transientes. Fonte: Elaborado pelo autor.

Por outro lado, estudamos os estados de sincronização para o mesmo conjunto de condições iniciais em cada iteração temporal, cuja configuração é dada por

$$
\left\{\begin{array}{l}
x_{1}(0)=0.05, \\
y_{1}(0)=0.20, \\
z_{1}(0)=0.02, \\
x_{2}(0)=-0.08, \\
y_{2}(0)=-0.10, \\
z_{2}(0)=-0.05,
\end{array}\right.
$$

então, observamos na Fig. 13 como o sistema Eq. 38 atinge um estado completo de sincronização para $\xi=1.0$, mostrando, portanto, que a configuração das condições iniciais organizadas para o sistema em estudo tem influência. 


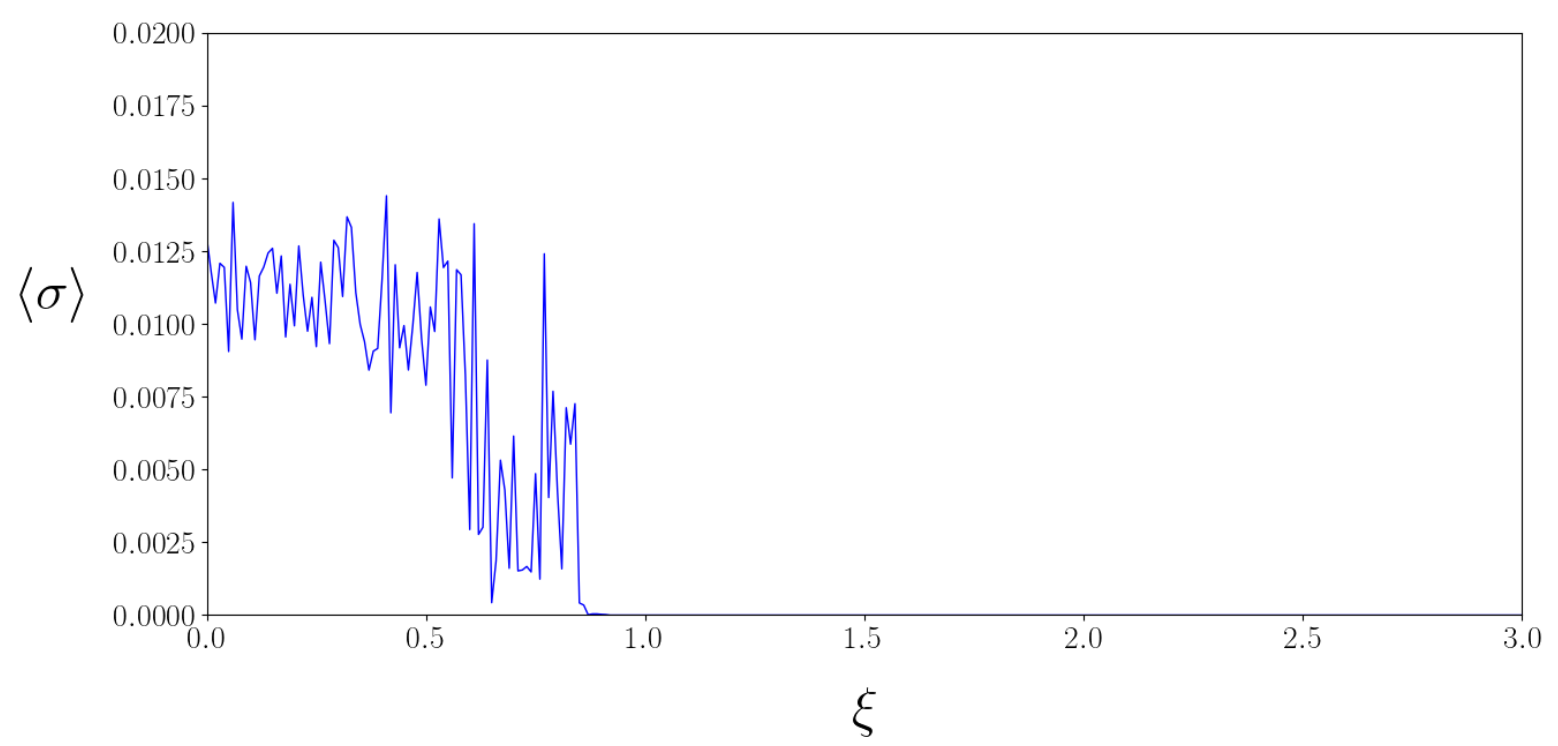

Figura 13 - Diagrama de sincronização do sistema Eq. (38) para uma rede de $N=2$ nós em função do parâmetro de acoplamento $\xi$ associado aos estados $x_{i}, y_{i}, z_{i}$ para a mesma condição inicial em cada iteração temporal; $\operatorname{com}(m, p, d, c, s, r)=$ $(0.02,0.4,1.0,50,10,0.1)$; acoplamento do tipo unidirecional. Os cálculos foram feitos com $5 \times 10^{4}$ iterações, ignorando $4 \times 10^{4}$ transientes e, portanto, considerando $1 \times 10^{4}$ iterados após os transientes. Fonte: Elaborado pelo autor.

Temos um segundo caso de acoplamento, que é dado pelo chamado acoplamento bidirecional, em que ambos os subsistemas são acoplados de tal forma que é possível que haja interação mútua entre as variáveis de estado de ambos os sistemas, então, temos que as equações que descrevem a dinâmica do sistema para um acoplamento bidirecional têm a forma

$$
\begin{aligned}
& \text { Subsistema } 1=\left\{\begin{array}{l}
\dot{x}_{1}=m y_{1}+p x_{1}\left(d-y_{1}^{2}\right), \\
\dot{y}_{1}=-x_{1}+c z_{1}, \\
\dot{z}_{1}=s x_{1}-r y_{1}+\xi\left(z_{2}-z_{1}\right),
\end{array}\right. \\
& \text { Subsistema } 2=\left\{\begin{array}{l}
\dot{x}_{2}=m y_{2}+p x_{2}\left(d-y_{2}^{2}\right), \\
\dot{y}_{2}=-x_{2}+c z_{2}, \\
\dot{z}_{2}=s x_{2}-r y_{2}+\xi\left(z_{1}-z_{2}\right),
\end{array}\right.
\end{aligned}
$$

onde $\xi_{1}=\xi_{2}=\xi$, e, portanto, há uma troca de informações entre os dois subsistemas. Assim, podemos analisar como está a evolução do sistema com essa configuração. Vemos na Fig. 14 que para condições iniciais que mudam em cada iteração temporal, o sistema alcança os estados de sincronização completa para um valor de $\xi=1.3$, sendo essa sincronização 
completa alcançada mais rapidamente do que para um acoplamento unidirecional, como visto na Fig. 12.

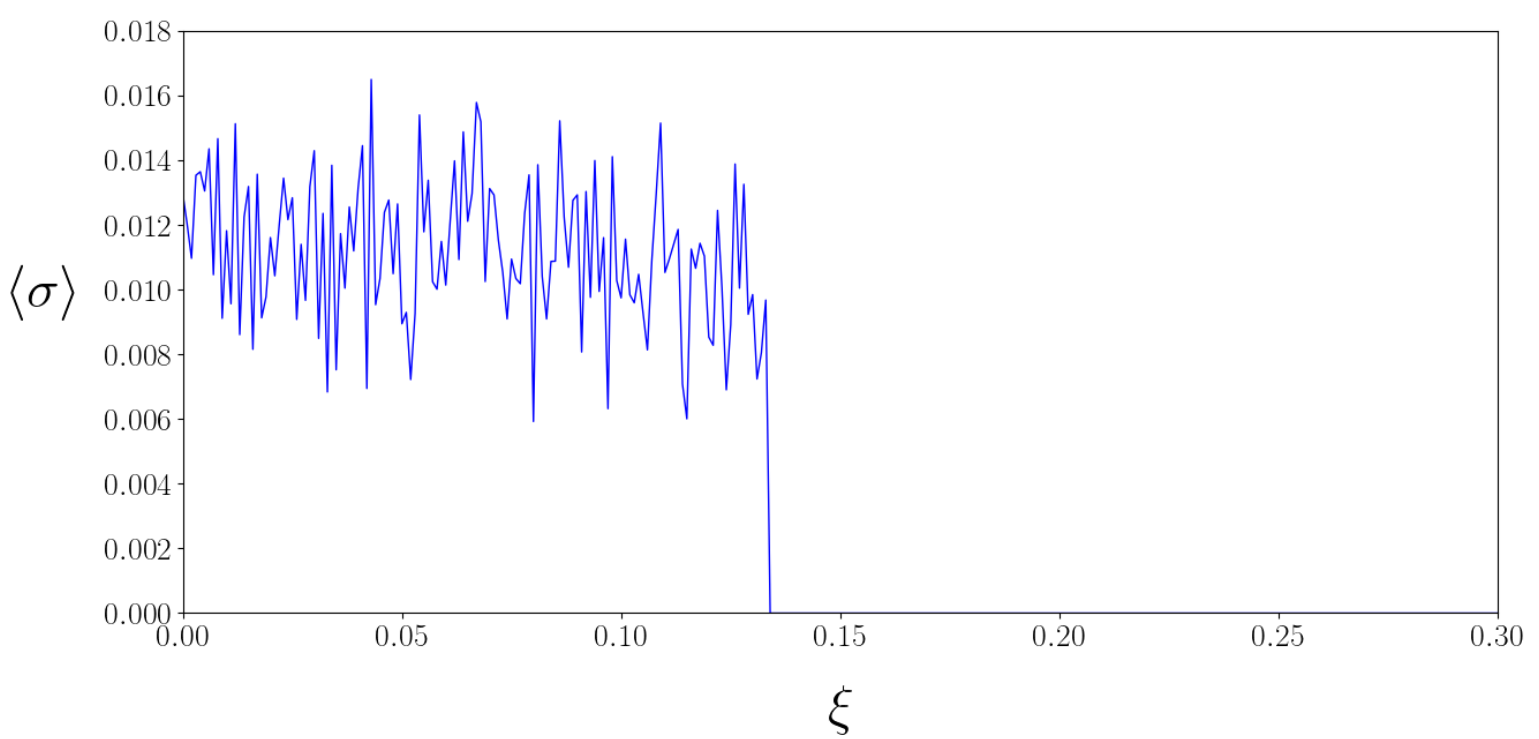

Figura 14 - Diagrama de sincronização do sistema Eq. (38) para uma rede de $N=2$ nós em função do parâmetro de acoplamento $\xi$ associado aos estados $x_{i}, y_{i}, z_{i}$ para diferentes condições iniciais em cada iteração temporal; $\operatorname{com}(m, p, d, c, s, r)=$ $(0.02,0.4,1.0,50,10,0.1)$; acoplamento do tipo bidirecional. Os cálculos foram feitos com $5 \times 10^{4}$ iterações, ignorando $4 \times 10^{4}$ transientes e, portanto, considerando $1 \times 10^{4}$ iterados após os transientes. Fonte: Elaborado pelo autor.

Agora, é muito interessante observar como quando o sistema exibe um comportamento coletivo complexo quando, em cada nova iteração temporária, ele assume as condições iniciais mostradas no Eq. 52. Vemos na Fig. 15 como o sistema Eq. 38 vai de uma dessincronização completa a uma sincronização "quase" incompleta entre o intervalo de valores $0.39 \lesssim \xi \lesssim 0.42$, e depois para um estado de sincronização completa que permanece entre os valores $0.43 \lesssim \xi \lesssim 1.48$, então, uma pequena intermitência é exibida para os valores de $\xi=1.49,1.50$, e depois estados de sincronização completa até $\xi=1.63$ onde o sistema dessincroniza completamente, mas rapidamente sua dinâmica converge para um ciclo limite. 


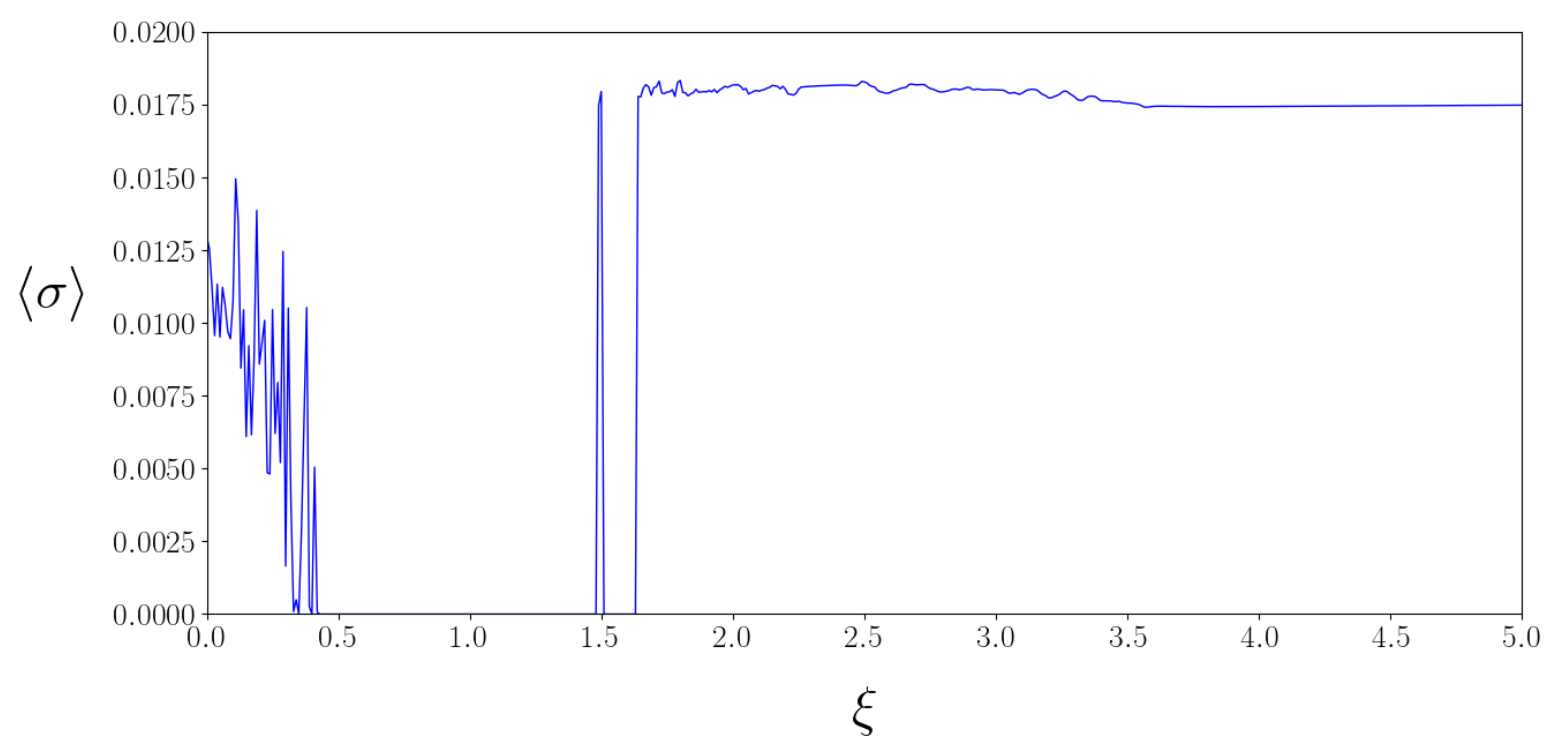

Figura 15 - Diagrama de sincronização do sistema Eq. (38) para uma rede de $N=2$ nós em função do parâmetro de acoplamento $\xi$ associado aos estados $x_{i}, y_{i}, z_{i}$ para a mesma condição inicial em cada iteração temporal; $\operatorname{com}(m, p, d, c, s, r)=$ $(0.02,0.4,1.0,50,10,0.1)$; acoplamento do tipo bidirecional. Os cálculos foram feitos com $5 \times 10^{4}$ iterações, ignorando $4 \times 10^{4}$ transientes e, portanto, considerando $1 \times 10^{4}$ iterados após os transientes. Fonte: Elaborado pelo autor.

\subsection{Análise de Estabilidade de Estados Sincronizados}

\subsubsection{Função Mestra de Estabilidade}

A macroeconomia modelada Eq. 38 é representada pelo seguinte vetor de estado

$$
\boldsymbol{\psi}_{i}(t)=\left[\begin{array}{l}
\psi_{i}^{1}(t) \\
\psi_{i}^{2}(t) \\
\psi_{i}^{3}(t)
\end{array}\right]=\left[\begin{array}{l}
x_{i}(t) \\
y_{i}(t) \\
z_{i}(t)
\end{array}\right]
$$

da mesma forma, a dinâmica de todos os osciladores é governada pelo campo vetorial $\mathbf{f}\left(\boldsymbol{\psi}_{i}(t)\right)$, cujo vetor é da forma

$$
\mathbf{f}\left(\boldsymbol{\psi}_{i}(t)\right)=\left[\begin{array}{c}
f_{1}\left(\boldsymbol{\psi}_{i}(t)\right) \\
f_{2}\left(\boldsymbol{\psi}_{i}(t)\right) \\
f_{3}\left(\boldsymbol{\psi}_{i}(t)\right)
\end{array}\right]=\left[\begin{array}{c}
m y_{i}+p x_{i}\left(d-y_{i}^{2}\right) \\
-x_{i}+c z_{i} \\
s x_{i}-r y_{i}
\end{array}\right]
$$


e o vetor de acoplamento $\mathbf{h}\left(\boldsymbol{\psi}_{i}(t)\right)$ para cada oscilador do sistema, é descrito por

$$
\mathbf{h}\left(\boldsymbol{\psi}_{i}(t)\right)=\left[\begin{array}{l}
h_{1}\left(\boldsymbol{\psi}_{i}(t)\right) \\
h_{2}\left(\boldsymbol{\psi}_{i}(t)\right) \\
h_{3}\left(\boldsymbol{\psi}_{i}(t)\right)
\end{array}\right]=\left[\begin{array}{c}
0 \\
0 \\
z_{i}
\end{array}\right],
$$

lembrando também que a matriz de acoplamento do nosso sistema corresponde à matriz Laplaciana $\boldsymbol{\Gamma}$, assim, para uma rede de $N=2$ osciladores acoplados, é dada por

$$
\boldsymbol{\Gamma}=\left[\begin{array}{rr}
1 & -1 \\
-1 & 1
\end{array}\right]
$$

tendo assim nossa topologia estabelecida. Vemos que os autovalores dessa matriz são dados por

$$
\operatorname{det}|\boldsymbol{\Gamma}-\lambda \mathbf{I}|=0
$$

sendo I a matriz identidade e cujos valores próprios são

$$
\left\{\begin{array}{l}
\lambda_{1}=0 \\
\lambda_{2}=2
\end{array}\right.
$$

e com seus respectivos autovetores, dados por

$$
v_{1}=\left[\begin{array}{l}
1 \\
1
\end{array}\right] ; \quad v_{2}=\left[\begin{array}{c}
1 \\
-1
\end{array}\right],
$$

onde podemos ver que o autovector $v_{1}$ nos diz que

$$
\boldsymbol{\psi}_{1}(t)=\boldsymbol{\psi}_{2}(t)
$$

correspondente à variedade de sincronismo idêntico, e o autovector $v_{2}$ implica

$$
\boldsymbol{\psi}_{1}(t)=-\boldsymbol{\psi}_{2}(t)
$$

sendo ortogonal a $v_{1}$ em um hiperplano formado por $\boldsymbol{\psi}_{1}(t) \times \boldsymbol{\psi}_{2}(t)$ (MONTEIRO, 2010). Portanto, a estabilidade do sistema é dada pela dinâmica gerada ao longo $v_{2}$. Lembre-se então que a dinâmica da rede de osciladores acoplada na variedade de sincronismo idêntico é

$$
\frac{d \boldsymbol{\psi}_{s}(t)}{d t}=\mathbf{f}\left(\boldsymbol{\psi}_{s}(t)\right)
$$


então, podemos escrever as variáveis de estado como

$$
\boldsymbol{\psi}_{s}(t)=\left[\begin{array}{l}
\psi_{s}^{1}(t) \\
\psi_{s}^{2}(t) \\
\psi_{s}^{3}(t)
\end{array}\right]=\left[\begin{array}{l}
x_{s}(t) \\
y_{s}(t) \\
z_{s}(t)
\end{array}\right]
$$

Agora a Equação mestra de estabilidade (Eq. 27) do sistema Macroeconômico Eq. 38 para $\boldsymbol{\psi}_{i}(t)=\boldsymbol{\psi}_{s}(t)$ é dada pela matriz

$$
\boldsymbol{P}_{i}(t)=\left[\begin{array}{lll}
\partial f_{1} / \partial x_{i}-\xi \mu_{i} \partial h_{1} / \partial x_{i} & \partial f_{1} / \partial y_{i}-\xi \mu_{i} \partial h_{1} / \partial y_{i} & \partial f_{1} / \partial z_{i}-\xi \mu_{i} \partial h_{1} / \partial z_{i} \\
\partial f_{2} / \partial x_{i}-\xi \mu_{i} \partial h_{2} / \partial x_{i} & \partial f_{2} / \partial y_{i}-\xi \mu_{i} \partial h_{2} / \partial y_{i} & \partial f_{2} / \partial z_{i}-\xi \mu_{i} \partial h_{2} / \partial z_{i} \\
\partial f_{3} / \partial x_{i}-\xi \mu_{i} \partial h_{3} / \partial x_{i} & \partial f_{3} / \partial y_{i}-\xi \mu_{i} \partial h_{3} / \partial y_{i} & \partial f_{3} / \partial z_{i}-\xi \mu_{i} \partial h_{3} / \partial z_{i}
\end{array}\right]
$$

substituindo assim cada uma das respectivas derivadas parciais, temos que

$$
\boldsymbol{P}_{i}(t)=\left[\begin{array}{ccc}
p\left(d-y_{i}^{2}\right) & m+2 p x_{i} y_{i} & 0 \\
-1 & 0 & c \\
s & -r & -\alpha
\end{array}\right]
$$

$\operatorname{com} \alpha=\xi_{i} \mu_{i}$. Assim, temos que para uma configuração de acoplamento unidireccional a matriz laplaciana é dada por

$$
\boldsymbol{\Gamma}=\left[\begin{array}{rr}
0 & 0 \\
-1 & 1
\end{array}\right]
$$

e os autovalores e autovetores

$$
\begin{gathered}
\left\{\begin{array}{l}
\mu_{1}=0, \\
\mu_{2}=1,
\end{array}\right. \\
\mathbf{v}_{\mu_{1}}=\left[\begin{array}{l}
0 \\
1
\end{array}\right] ; \mathbf{v}_{\mu_{2}}=\left[\begin{array}{l}
1 \\
1
\end{array}\right] .
\end{gathered}
$$

Sabemos que o maior valor próprio $\mu_{2}=1$, está associado a $\boldsymbol{P}_{2}(t)$, portanto, temos que a evolução temporal da dinâmica transversal do sistema é

$$
\dot{\boldsymbol{\delta}}_{2}(t)=\boldsymbol{P}_{2}(t) \boldsymbol{\delta}_{2}(t) .
$$

Agora como a matriz $\boldsymbol{P}_{i}(t)$ não contém equações lineares, devemos integrar numericamente para encontrar sua solução. Para integrar numericamente essas equações, escrevemos

$$
\boldsymbol{\delta}_{2}(t)=\left[\begin{array}{l}
\delta_{2}^{1}(t) \\
\delta_{2}^{2}(t) \\
\delta_{2}^{3}(t)
\end{array}\right]=\left[\begin{array}{l}
\delta x(t) \\
\delta y(t) \\
\delta z(t)
\end{array}\right]
$$


e então nós temos que

$$
\left[\begin{array}{l}
\dot{\delta} x(t) \\
\dot{\delta} y(t) \\
\dot{\delta} z(t)
\end{array}\right]=\left[\begin{array}{ccc}
p\left(d-y_{i}^{2}\right) & m+2 p x_{i} y_{i} & 0 \\
-1 & 0 & c \\
s & -r & -\alpha
\end{array}\right]=\left[\begin{array}{c}
\delta x(t) \\
\delta y(t) \\
\delta z(t)
\end{array}\right],
$$

agora partimos do conjunto de condições iniciais para as perturbações

$$
\boldsymbol{\delta}_{2}(t)=\left[\begin{array}{l}
\delta x(t) \\
\delta y(t) \\
\delta z(t)
\end{array}\right]=\left[\begin{array}{l}
0 \\
0 \\
1
\end{array}\right]
$$

Logo, integramos ambas as equações $\boldsymbol{\psi}_{s}(t)$ e $\boldsymbol{\delta}_{2}(t)$ pelo método Runge-Kutta de quarta ordem (RK4) Veja Apêndice A. Agora, podemos observar na Fig. 16, a simulação numérica para a dinâmica transversal do sistema Eq. 38 para um acoplamento unidirecional fornecido pela condição Eq. 76 onde o maior expoente de Lyapunov $\lambda_{T}$ associado a $\boldsymbol{P}_{2}(t)$ dependendo do parâmetro de acoplamento $\xi_{2}=\xi$ é observado. Podemos ver como o sistema inicia em $\lambda_{T}=0.26$ permanece positivo, portanto sua sincronização é instável, até atingir um valor crítico de $\xi_{c}^{1}=1.5$, onde o sistema é negativo, convergindo para a variedade de sincronismo idêntico e atingindo seu valor máximo de estabilidade, dado um ponto mínimo de $\lambda_{T}=-0.369$, então, o sistema começa a sair da estabilidade a ponto de ficar instável novamente por um segundo valor crítico $\xi_{c}^{2}=19.3$, onde sua instabilidade é mantida por valores mais altos, chegando a $k_{1}=100$. 


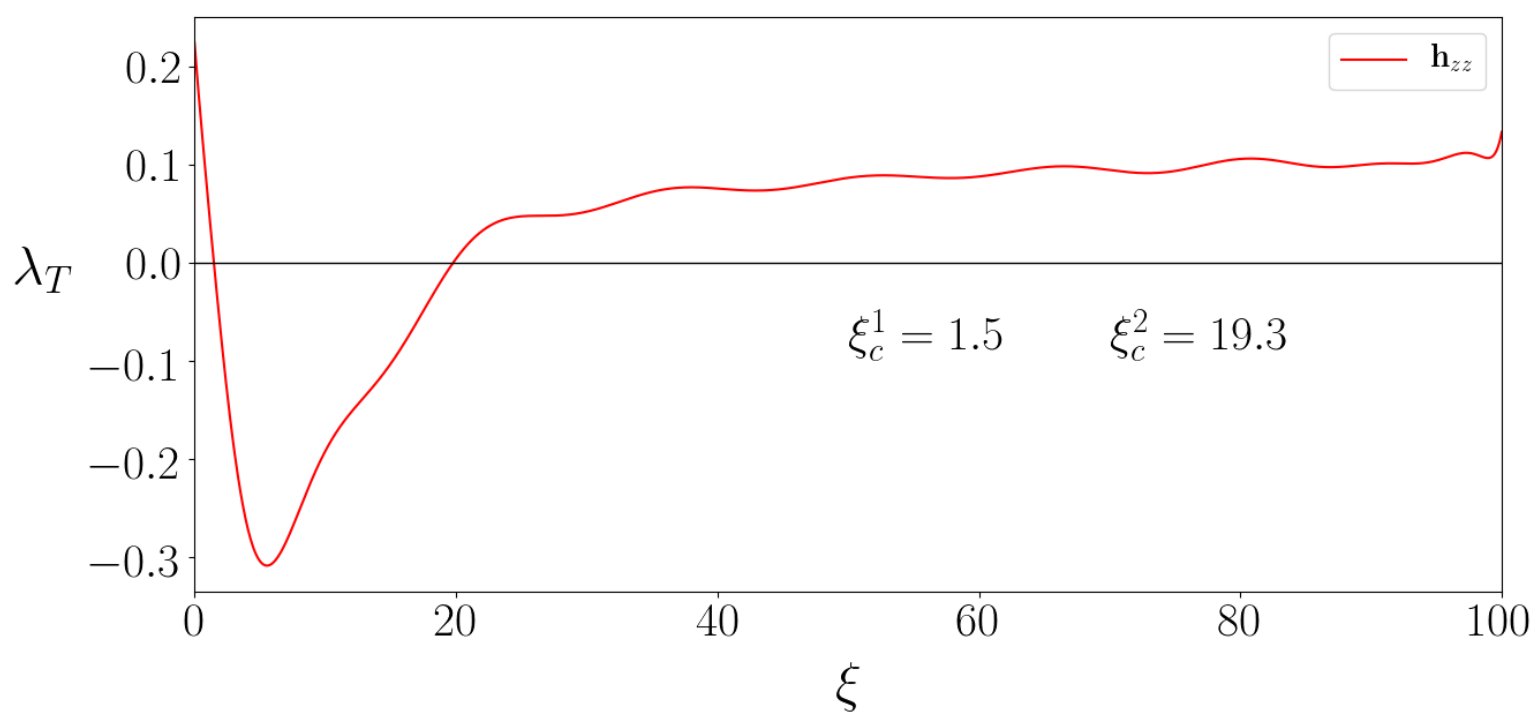

Figura 16 - Função Mestra de Estabilidade $\lambda_{T}$ em função do parâmetro de acoplamento $\xi$ para o sistema Eq. 38 com uma configuração unidireccional; fixando os valores $(N, m, p, d, c, s, r)=(2,0.02,0.4,1.0,50,10,0.1)$. Fonte: Elaborado pelo autor.

É possível calcular todos os acoplamentos possíveis, um por um do sistema, simplesmente variando a função de acoplamento $\mathbf{h}(\boldsymbol{\psi}(t))$,

$$
\mathbf{h}(\boldsymbol{\psi}(t))=\left[\begin{array}{l}
h_{1}(\boldsymbol{\psi}(t)) \\
h_{2}(\boldsymbol{\psi}(t)) \\
h_{3}(\boldsymbol{\psi}(t))
\end{array}\right]=\left[\begin{array}{l}
x(t) \\
y(t) \\
z(t)
\end{array}\right] .
$$

As nove possibilidades de acoplamento um a um podem ser vistas na Fig. 17, que mostra o diagrama das funções mestras de estabilidade para os diferentes tipos de acoplamentos. Podemos observar como o sistema atinge estados de estabilidade em sua sincronização apenas para os acoplamentos $y(t) \rightarrow y(t), z(t) \rightarrow z(t)$ e $z(t) \rightarrow x(t)$. Para um acoplamento $y(t) \rightarrow y(t)$ temos uma faixa de estabilidade entre os valores críticos de $\xi_{c}^{1}=1.5$ e $\xi_{c}^{1}=19.7$, agora, para a configuração $z(t) \rightarrow z(t)$ temos uma gama de estabilidade entre os valores críticos de $\xi_{c}^{1}=1.2$ e $\xi_{c}^{1}=19.3$ e finalmente temos que, para o acoplamento $z(t) \rightarrow x(t)$ estabilidade é obtida em uma faixa de valores críticos de $\xi_{c}^{1}=1.6$ y $\xi_{c}^{1}=5.6$. 

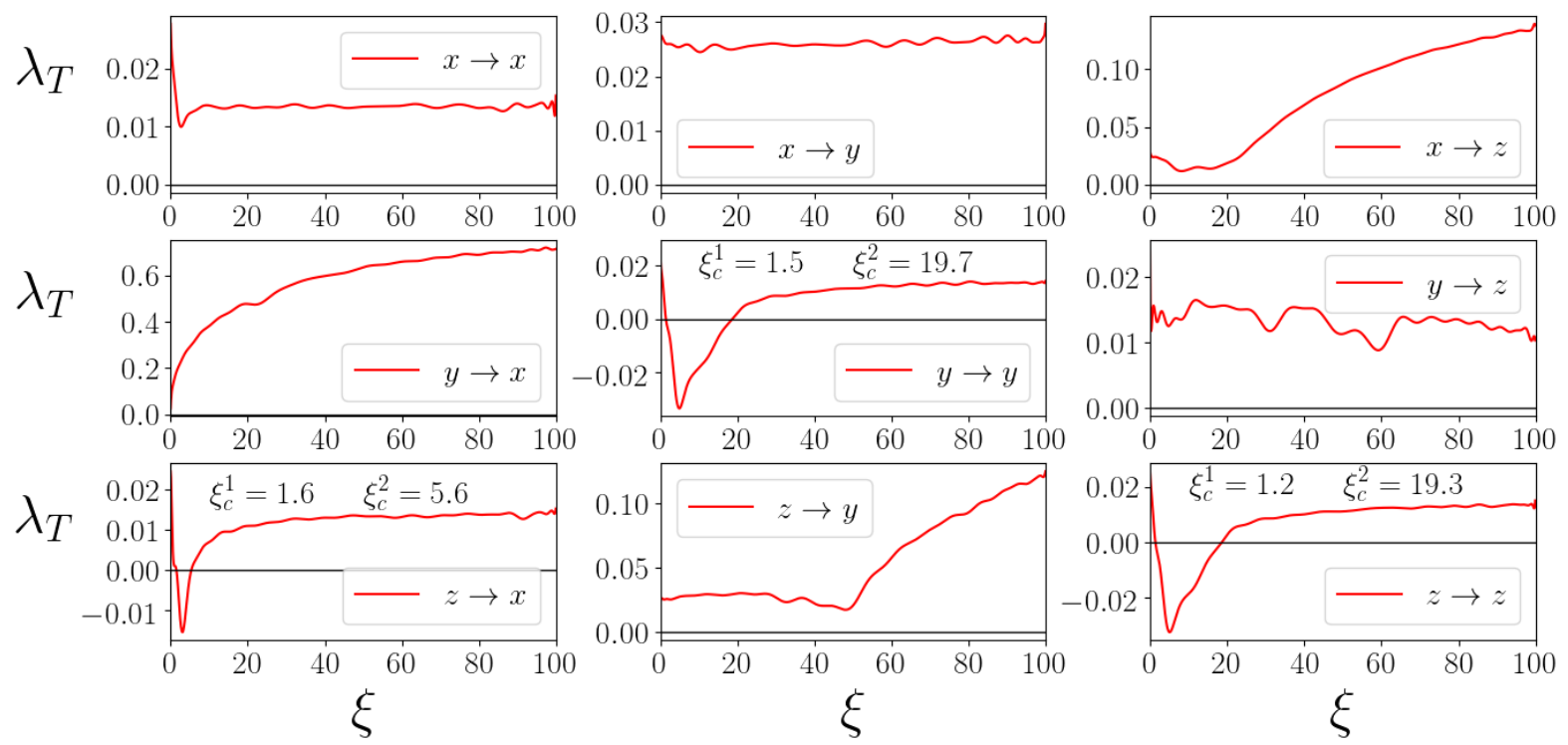

Figura 17 - Função Mestra de Estabilidade $\lambda_{T}$ sob vários esquemas de acoplamento $\xi$ para o sistema Eq. 38 com uma configuração unidireccional; fixando os valores $(N, m, p, d, c, s, r)=(2,0.02,0.4,1.0,50,10,0.1)$. A notação $x, y, z \rightarrow x, y, z$, indica o acoplamento como sendo dos componentes $x, y, z$ de um oscilador para os componentes $x, y, z$ de outro oscilador.Fonte: Elaborado pelo autor.

Agora, executamos o mesmo procedimento, mas para uma configuração de acoplamento bidirecional que é fornecido por a matriz laplaciana dada por

$$
\boldsymbol{\Gamma}=\left[\begin{array}{rr}
1 & -1 \\
-1 & 1
\end{array}\right]
$$

e os autovalores e autovetores

$$
\begin{gathered}
\left\{\begin{array}{l}
\mu_{1}=0, \\
\mu_{2}=2,
\end{array}\right. \\
\mathbf{v}_{\mu_{1}}=\left[\begin{array}{l}
1 \\
1
\end{array}\right] ; \mathbf{v}_{\mu_{2}}=\left[\begin{array}{c}
1 \\
-1
\end{array}\right],
\end{gathered}
$$

assim, os dois subsistemas trocam informações entre si com a mesma intensidade de acoplamento. Vemos na Fig. 18 que agora o sistema Eq. 38 da mesma maneira que para o sistema com acoplamento unidirecional, ele começa em $\lambda_{T}=0.26$ e converge rapidamente para um estado de estabilidade por um valor crítico de $\xi_{c}=0.2$, mas, diferentemente do acoplamento unidirecional observado anteriormente, o máximo expoente de Lyapunov permanece na região negativa que corresponde à estabilidade do sistema. 


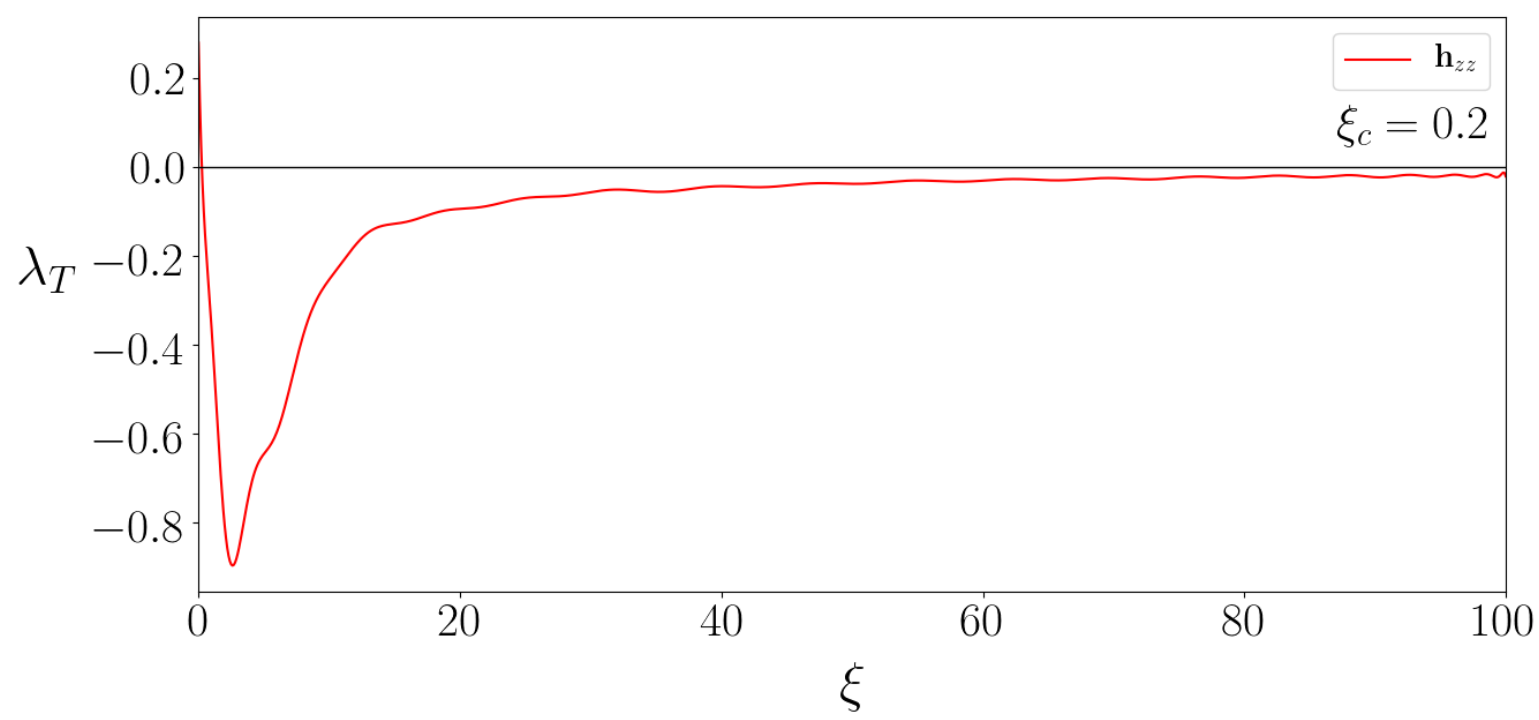

Figura 18 - Função Mestra de Estabilidade $\lambda_{T}$ em função do parâmetro de acoplamento $\xi$ para o sistema Eq. 38 com uma configuração bidireccional; fixando os valores $(N, m, p, d, c, s, r)=(2,0.02,0.4,1.0,50,10,0.1)$. Fonte: Elaborado pelo autor.

Da mesma forma, para cada um dos nove tipos de acoplamentos um a um, podemos ver na Fig. 19 particularmente para as configurações $y(t) \rightarrow y(t), z(t) \rightarrow z(t)$ e $z(t) \rightarrow x(t)$, que o sistema permanece estável, diferente do observado na Fig. 17 para um acoplamento unidirecional. Isso nos mostra que a troca de informações entre os dois subsistemas permite gerar maior estabilidade na rede de osciladores acoplados. 

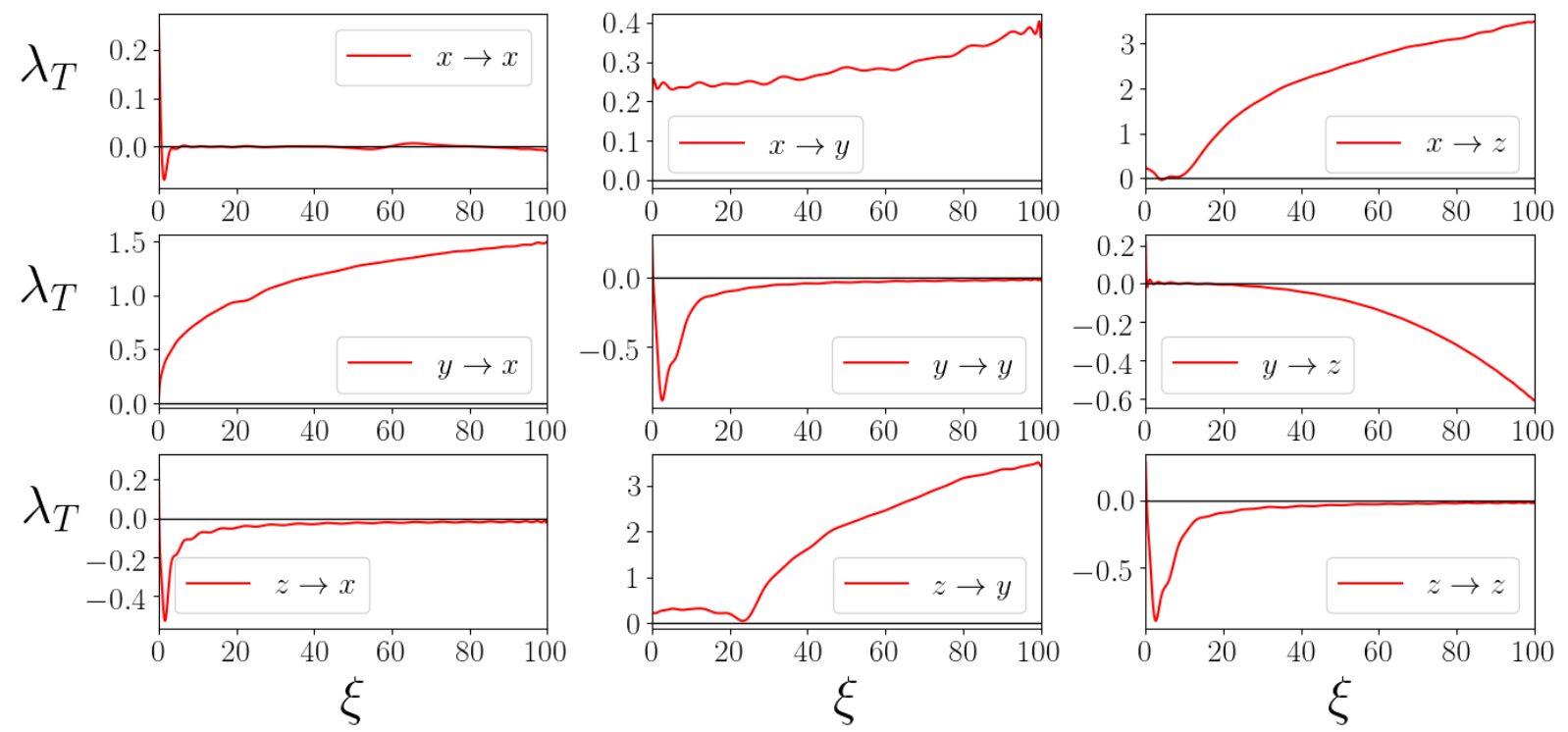

Figura 19 - Função Mestra de Estabilidade $\lambda_{T}$ sob vários esquemas de acoplamento $\xi$ para o sistema Eq. 38; com $(N, m, p, d, c, s, r)=(2,0.02,0.4,1.0,50,10,0.1)$. A notação $x, y, z \rightarrow x, y, z$, indica o acoplamento como sendo dos componentes $x, y, z$ de um oscilador para os componentes $x, y, z$ de outro oscilador.Fonte: Elaborado pelo autor.

Finalmente, podemos observar na Fig. 20 como, se fizermos um acoplamento em todas as variáveis macroeconômicas do sistema da forma

$$
\mathbf{h}_{\text {xxyyzz }}=\left[\begin{array}{l}
h_{1}(\boldsymbol{\psi}(t)) \\
h_{2}(\boldsymbol{\psi}(t)) \\
h_{3}(\boldsymbol{\psi}(t))
\end{array}\right]=\left[\begin{array}{l}
x(t) \\
y(t) \\
z(t)
\end{array}\right] .
$$

na rede de osciladores acoplados, é gerada uma maior estabilidade de sincronização, em particular, atinge a estabilidade por um valor crítico de $\xi_{c}^{1}=0.1$. Comparamos para um tipo de acoplamento da forma

$$
\mathbf{h}_{y y z z}=\left[\begin{array}{c}
h_{1}(\boldsymbol{\psi}(t)) \\
h_{2}(\boldsymbol{\psi}(t)) \\
h_{3}(\boldsymbol{\psi}(t))
\end{array}\right]=\left[\begin{array}{c}
0 \\
y(t) \\
z(t)
\end{array}\right],
$$

onde a sincronização é estável a partir de um valor crítico de $\xi_{c}^{2}=0.2$, e para um tipo de acoplamento individual

$$
\mathbf{h}_{z z}=\left[\begin{array}{c}
h_{1}(\boldsymbol{\psi}(t)) \\
h_{2}(\boldsymbol{\psi}(t)) \\
h_{3}(\boldsymbol{\psi}(t))
\end{array}\right]=\left[\begin{array}{c}
0 \\
0 \\
z(t)
\end{array}\right],
$$


onde o sistema estabiliza para um valor crítico de $\xi_{c}^{2}=0.3$.

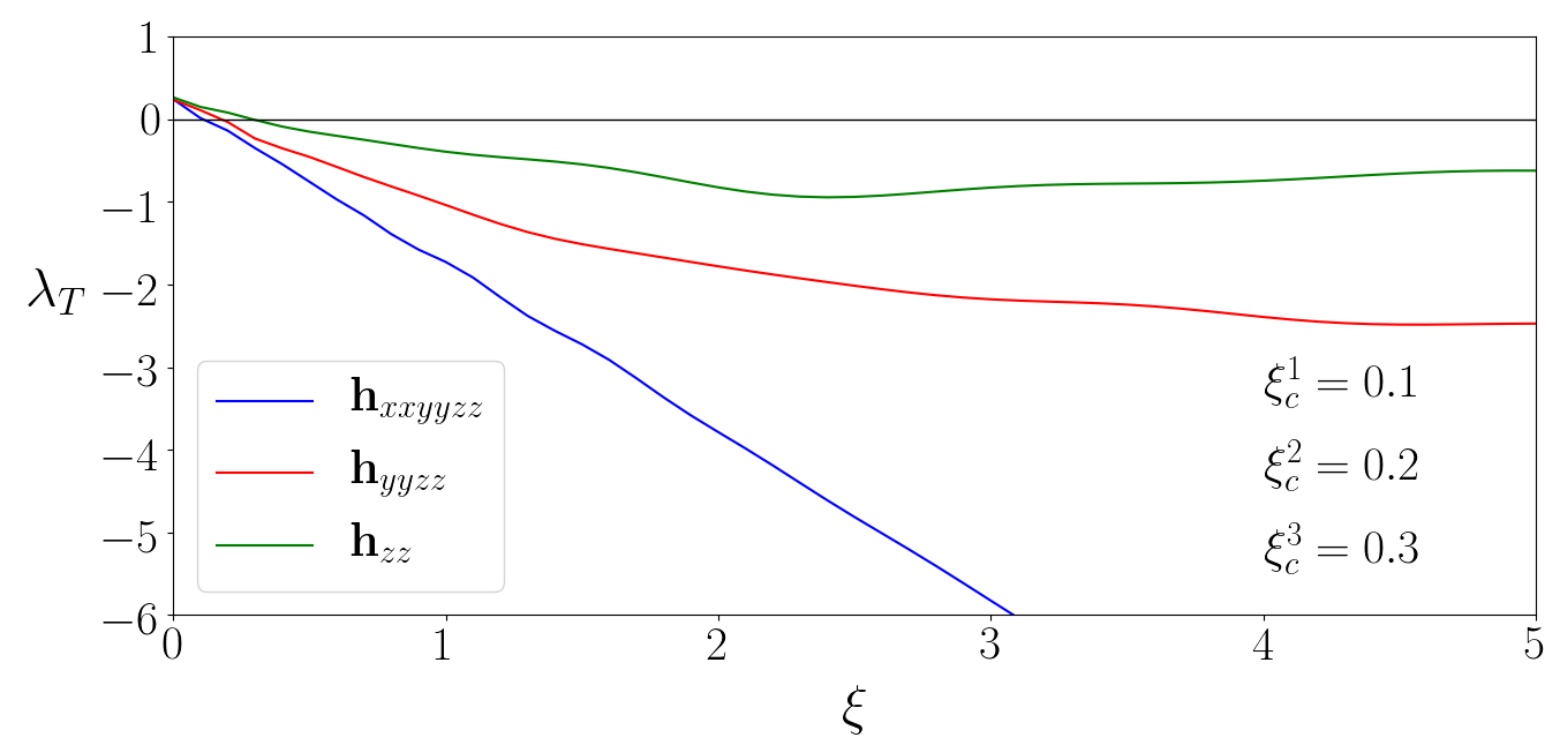

Figura 20 - Função Mestra de Estabilidade $\lambda_{T}$ acoplada por meio de $\xi$ pelos diferentes componentes $z \rightarrow z, y, z \rightarrow y, z$ e $x, y, z \rightarrow x, y, z$ para o sistema Eq. 38; com $(N, m, p, d, c, s, r)=(2,0.02,0.4,1.0,50,10,0.1)$. Fonte: Elaborado pelo autor. 


\section{Conclusão}

As economias (Eq. 38) foram acopladas por meio dos parâmetros de controle $\xi_{i}$. Preliminarmente, consideramos o caso em que há apenas duas economias, sendo uma a 'mestre' $\xi_{1}=0$ e a outra $\xi_{2}=\xi>0$ a 'escrava', também chamado de modelo unidirecional (VOLOS; KYPRIANIDIS; STOUBOULOS, 2012). Neste caso, a economia 1 exerce influência sobre a economia 2, mas não o inverso. Por meio da análise da função de estabilidade mestra $\lambda_{T}$ pudemos estudar a estabilidade da sincronização deste tipo de acoplamento, reportado na figura Fig. 16 . Inicialmente, observamos que não ocorre sincronização para baixos valores de $\xi$, uma vez que os parâmetros escolhidos colocam os subsistemas na fase caótica e ainda a força do acoplamento não é capaz de promover a sincronzação. O aumento no valor do parâmetro de controle leva à sincronização estável do sistema. No entanto, com o aumento do valor de $\xi>15$ o sistema desestabiliza, retornando a estabilidade no intervalo de $\xi=(35$ a 38). Para valores superiores, no intervalo estudado de $\xi=(39$ a 100) o sistema perde a estabilidade. No caso bidirecional, o sistema entra na fase estável e não se observou a volta para o regime instável (Fig. 18). Apesar de que cada economia (subsistema) seja intrinsecamente caótica, o acoplamento difusivo do modelo permite a emergência da sincronização de forma estável.

O modelo macroeconômico aqui descreve a relação entre poupança, PIB e entrada de capital estrangeiro. Ele é bem rudimentar, e possui premissas que podem ser questionadas pelos economistas e possivelmente melhoradas. A equação que descreve a variação da poupança depende da variação do PIB, e concomitantemente a poupança cresce com o aumento do PIB, enquanto a condição $b-y^{2}>0$ for satisfeita. Caso contrário, o nível de poupança diminui, o que pode significar um aumento no consumo e uma pressão para o incremento da inflação. O PIB também é afetado pelo nível de poupança e pela entrada de capital externo. Contudo, isso leva à possibilidade de uma influência do exterior sobre a economia nacional via fluxo de entrada de capital $(z)$. Notamos que as economias podem atuar de forma cooperativa e ter como resultado coletivo a sincronização entre as variáveis macroeconômicas, simplesmente pelo ajuste do parâmetro de controle. O comportamento oscilatório das variáveis macroeconômicas emula os ciclos econômicos, mais ainda, as flutuações são endógenas, emergentes, podendo ser auto-sustentáveis ou caóticas. Neste modelo, os períodos de oscilação dependem do valor de $\xi_{i}$. Em dados empíricos, esses 
períodos podem variar de poucos anos até decadas. Assim, ao introduzir ruídos no modelo permitirá reproduzir cenários mais realísticos. Porém, também vai demandar o uso de alguns métodos, entre eles o da Função Mestra de Estabilidade que permitira tirar conclusões mais realistas. Nosso foco não foi apresentar um modelo macroeconômico realista, mas sim, operar uma técnica de análise da estabilidade da sincronização entre economias acopladas. Avançamos em relação ao trabalho de Volos et al. (VOLOS; KYPRIANIDIS; STOUBOULOS, 2012) que discutiram o caso de apenas duas economias acopladas. Muito embora eles tenham demostrado que este sistema (de duas economias) podem sincronizar, faltou discutir se os estados sincronizados são estáveis. A fim de estender a análise para uma rede de economias com um número arbitrário de paises $N$ optamos por usar a função de estabilidade mestra. Neste trabalho apresentamos a versão generalizada do modelo de Bouali (BOUALI, 1999; VOLOS; KYPRIANIDIS; STOUBOULOS, 2012) em uma rede acoplada. Verificamos que este modelo encapsula uma riqueza de comportamentos dinâmicos complexos que permite estudar vários regimes tais como os ciclos econômicos ou cenários aperiódicos e instáveis. Uma versão simplificada como a apresentada aqui é importante para endereçar questões específicas que considere efeitos globais e influências mútuas, simétricas e assimétricas de economias acopladas em redes. A contribuição deste trabalho foi aplicar o método da Função Mestra de Estabilidade para o estudo de uma rede de sistemas que representa qualitativamente uma macroeconomia. Como sugestão de trabalhos futuros podemos estudar topologias de redes mais realistas, fazer um estudo mais detalhado do efeito da variação dos parâmetros de modelo generalizado e ainda confrontar com dados empíricos. De forma geral, o método de análise pode ser aplicado para o estudo de modelos dinâmicos não lineares mais sofisticados. 


\section{Referências}

BARNETT, W. A.; DUZHAK, E. A. Empirical assessment of bifurcation regions within new keynesian models. Economic Theory, Springer, v. 45, n. 1-2, p. 99-128, 2010. Citado na página 20.

BOUALI, S. Feedback loop in extended van der pol's equation applied to an economic model of cycles. International Journal of Bifurcation and Chaos, World Scientific, v. 9, n. 04, p. 745-756, 1999. Citado 3 vezes nas páginas 11, 21 e 53.

BOUALI, S.; BUSCARINO, A.; FORTUNA, L.; FRASCA, M.; GAMBUZZA, L. Emulating complex business cycles by using an electronic analogue. Nonlinear Analysis: Real World Applications, Elsevier, v. 13, n. 6, p. 2459-2465, 2012. Citado na página 20.

CALDERóN, C.; FUENTES, J. R. Have business cycles changed over the last two decades? an empirical investigation. Journal of Development Economics, Elsevier, v. 109, p. 98-123, 2014. Citado na página 20.

CARTWRiGHT, J. H.; EGUÍluZ, V. M.; HERNÁNDEZ-GARCÍA, E.; PIRO, O. Dynamics of elastic excitable media. International Journal of Bifurcation and Chaos, World Scientific, v. 9, n. 11, p. 2197-2202, 1999. Citado na página 19.

FLAHERTY, J. E.; HOPPENSTEADT, F. Frequency entrainment of a forced van der pol oscillator. Studies in Applied Mathematics, Wiley Online Library, v. 58, n. 1, p. 5-15, 1978. Citado na página 19.

FREDERICKSON, P.; KAPLAN, J. L.; YORKE, E. D.; YORKE, J. A. The liapunov dimension of strange attractors. Journal of differential equations, Elsevier, v. 49, n. 2, p. 185-207, 1983. Citado na página 28.

GARDNER, T.; CECCHI, G.; MAGNASCO, M.; LAJE, R.; MINDLIN, G. B. Simple motor gestures for birdsongs. Physical review letters, APS, v. 87, n. 20, p. 208101, 2001. Citado na página 19.

GOODWIN, P. The control of branch growth on potato tubers: Ii. the pattern of sprout growth. Journal of Experimental Botany, Oxford University Press, v. 18, n. 1, p. 87-99, 1967. Citado na página 20.

GYERGYEK, T.; ČERČEK, M.; STANOJEVIĆ, M. On the nonlinear dynamics of potential relaxation instability in a weakly magnetized discharge plasma. Open Systems $\mathscr{6}$ Information Dynamics, Springer, v. 4, n. 4, p. 479-486, 1997. Citado na página 19.

HUANG, L.; CHEN, Q.; LAI, Y.-C.; PECORA, L. M. Generic behavior of master-stability functions in coupled nonlinear dynamical systems. Physical Review E, APS, v. 80, n. 3, p. 036204, 2009. Citado 2 vezes nas páginas 14 e 22.

HUYGENS, C.; OSCILLATORIUM, H. The pendulum clock. Trans RJ Blackwell, The Iowa State University Press, Ames, Springer, 1986. Citado na página 33.

KAPLAN, J. L.; YORKE, J. A. Chaotic behavior of multidimensional difference equations. In: Functional differential equations and approximation of fixed points. [S.1.]: Springer, 1979. p. 204-227. Citado na página 28. 
KROGDAHL, W. S. Stellar pulsation as a limit-cycle phenomenon. The Astrophysical Journal, v. 122, p. 43, 1955. Citado na página 19.

KYRTSOU, C.; VORLOW, C. E. Complex dynamics in macroeconomics: A novel approach. In: New Trends in Macroeconomics. [S.l.]: Springer, 2005. p. 223-238. Citado na página 20.

LIU, Y.; LI, R.; CHE, Y.; HAN, C. Chaos synchronization of coupled neurons via h-infinity control with cooperative weights neural network. In: Advances in Future Computer and Control Systems. [S.l.]: Springer, 2012. p. 369-374. Citado na página 33.

LYAPUNOV, A. M. Reports of kharkov. Math. Soc. Ser, v. 2, n. 4, p. 1-2, 1893. Citado na página 27.

LYAPUNOV, A. M.; WALKER, J. The general problem of the stability of motion. [S.l.]: American Society of Mechanical Engineers Digital Collection, 1994. Citado na página 27.

MONTEIRO, L. H. A. Sistemas dinâmicos complexos. Livraria da Física, São Paulo, 2010. Citado 4 vezes nas páginas 14, 20, 22 e 43.

PASSOS, D.; LOPES, I. A low-order solar dynamo model: inferred meridional circulation variations since 1750. The Astrophysical Journal, IOP Publishing, v. 686, n. 2, p. 1420, 2008. Citado na página 19.

PECORA, L.; CARROLL, T.; JOHNSON, G.; MAR, D.; FINK, K. S. Synchronization stability in coupled oscillator arrays: Solution for arbitrary configurations. International Journal of Bifurcation and Chaos, World Scientific, v. 10, n. 02, p. 273-290, 2000. Citado na página 14.

PECORA, L. M.; CARROLL, T. L. Synchronization in chaotic systems. Physical review letters, APS, v. 64, n. 8, p. 821, 1990. Citado na página 9.

PECORA, L. M.; CARROLL, T. L. Driving systems with chaotic signals. Physical Review $A$, APS, v. 44, n. 4, p. 2374, 1991. Citado na página 9.

PECORA, L. M.; CARROLL, T. L. Master stability functions for synchronized coupled systems. Physical review letters, APS, v. 80, n. 10, p. 2109, 1998. Citado 2 vezes nas páginas 18 e 22.

PIKOVSKY, A.; KURTHS, J.; ROSENBLUM, M.; KURTHS, J. Synchronization: a universal concept in nonlinear sciences. [S.l.]: Cambridge university press, 2003. v. 12. Citado na página 33.

POL, B. V. D.; MARK, J. V. D. Lxxii. the heartbeat considered as a relaxation oscillation, and an electrical model of the heart. The London, Edinburgh, and Dublin Philosophical Magazine and Journal of Science, Taylor \& Francis, v. 6, n. 38, p. 763-775, 1928. Citado na página 19.

POL, B. Van der. Lxxxviii. on "relaxation-oscillations". The London, Edinburgh, and Dublin Philosophical Magazine and Journal of Science, Taylor \& Francis, v. 2, n. 11, p. 978-992, 1926. Citado 2 vezes nas páginas 11 e 19. 
ROWAT, P. F.; SELVERSTON, A. I. Modeling the gastric mill central pattern generator of the lobster with a relaxation-oscillator network. Journal of neurophysiology, American Physiological Society Bethesda, MD, v. 70, n. 3, p. 1030-1053, 1993. Citado na página 19.

SHAHVERDIEV, E.; SHORE, K. Synchronization of chaos in unidirectionally and bidirectionally coupled multiple time delay laser diodes with electro-optical feedback. Optics Communications, Elsevier, v. 282, n. 2, p. 310-316, 2009. Citado na página 33.

SPROTT, J. Dynamical models of happiness. Nonlinear Dynamics, Psychology, and Life Sciences, Citeseer, v. 9, n. 1, p. 23-36, 2005. Citado na página 19.

VOLOS, C. K.; KYPRIANIDIS, I. M.; STOUBOULOS, I. N. Synchronization phenomena in coupled nonlinear systems applied in economic cycles. WSEAS Trans. Syst, v. 11, n. 12, p. 681-690, 2012. Citado 4 vezes nas páginas 20, 21, 52 e 53.

WOLF, A.; SWIFT, J. B.; SWINNEY, H. L.; VASTANO, J. A. Determining lyapunov exponents from a time series. Physica D: Nonlinear Phenomena, Elsevier, v. 16, n. 3, p. 285-317, 1985. Citado na página 21. 


\section{Apêndice A - Runge-Kutta 4to ordem}

O método de Runge-Kutta de quarta ordem é obtido a partir da série de Taylor ao longo das mesmas linhas que o método de segunda ordem. Como a derivação é bastante longa e não muito instrutiva, devemos ignorá-la. A forma final da fórmula de integração novamente depende da escolha dos parâmetros; isto é, não existe uma fórmula única de quarta ordem de Runge-Kutta. A versão mais popular, conhecida simplesmente como método Runge-Kutta, envolve a seguinte sequência de operações

$$
\begin{aligned}
\mathbf{K}_{0} & =h \mathbf{F}(x, y) \\
\mathbf{K}_{1} & =h \mathbf{F}\left(x+\frac{h}{2}, y+\frac{\mathbf{K}_{0}}{2}\right) \\
\mathbf{K}_{2} & =h \mathbf{F}\left(x+\frac{h}{2}, y+\frac{\mathbf{K}_{1}}{2}\right) \\
\mathbf{K}_{3} & =h \mathbf{F}\left(x+h, y+\mathbf{K}_{2}\right) \\
y(x+h) & =y(x)+\frac{1}{6}\left(\mathbf{K}_{0}+2 \mathbf{K}_{1}+2 \mathbf{K}_{2}+\mathbf{K}_{3}\right)
\end{aligned}
$$

